\title{
Frequency Modulated Continuous Wave Radar System at ISM Band for Short Range Indoor Positioning
}

\author{
A Thesis \\ Presented to \\ the Faculty of the School of Engineering and Applied Science \\ University of Virginia
}

\author{
In Partial Fulfillment \\ of the Requirements for the Degree \\ Master of Science in Electrical Engineering
}

By

Yuxin Wang 


\section{Abstract}

Frequency modulated continuous wave $(\mathrm{FMCW})$ radar is a technique for obtaining range information from a radar by frequency modulating a continuous signal. The frequency range of FMCW radar varies from $900 \mathrm{MHz}$ to $80 \mathrm{GHz}$, and one of the applications is human positioning for indoor healthcare scenarios and intelligent housing system. FMCW radar system is becoming more portable, more precise and cheaper. In this thesis, a FMCW radar system at $2.4 \mathrm{GHz}$ ISM band is proposed for intelligent housing system.

The thesis proposes an economic and efficient DDS-based PLL frequency synthesizer. It can generate a frequency modulated continuous wave from $2.4 \mathrm{GHz}$ to $2.5 \mathrm{GHz}$ in $1 \mathrm{~ms}$. The thesis uses the ADF4158 phase locked loop (PLL) chip to implement triangle frequency modulation in order to generate the $2.4 \mathrm{GHz}$ continuous wave signal, and in this frequency synthesizer system, a direct digital synthesizer (DDS) chip is used, as the reference frequency of PLL, to analyze crystalbased PLL and DDS based PLL. The PLL frequency synthesizer shows high accuracy and low phase noise, and it is easy to be controlled by PC.

The FMCW radar system adopts a homodyne transceiver architecture. The thesis shows effective design of each part of the homodyne receiver, including amplifiers, filters and a mixer. The whole transceiver shows the performance in position estimation and tracking objects within 10 meters with less than $2 \%$ error. 


\section{Contents}

1 Introduction 1

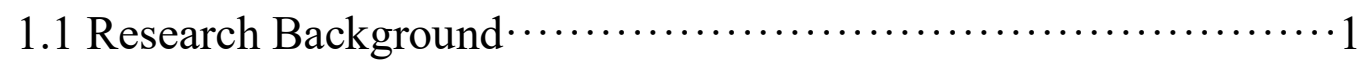

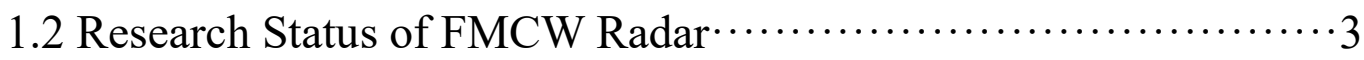

1.3 Research Status of PLL Frequency Synthesizer $\cdots \cdots \cdots \cdots \cdots \cdots \cdots \cdots$

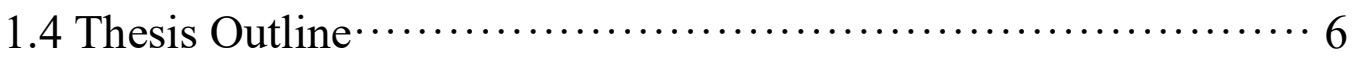

2 FMCW Radar Theory 7

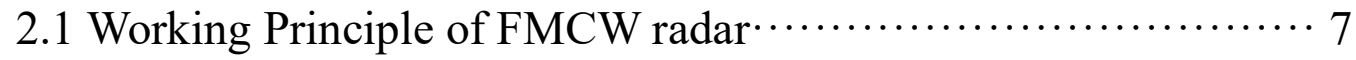

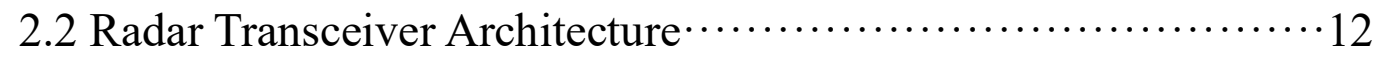

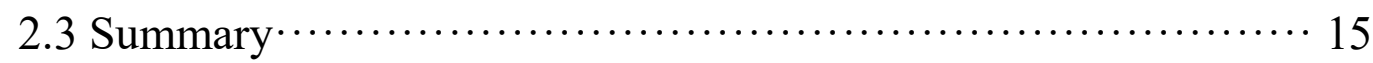

3 DDS-based PLL Frequency Synthesizer 16

3.1 Working Principle of Direct Digital Synthesizer $\cdots \cdots \cdots \cdots \cdots \cdots \cdots \cdots+\cdots$

3.2 Phase-locked Loop Frequency Synthesizer $\cdots \cdots \cdots \cdots \cdots \cdots \cdots \cdots+\cdots \cdots \cdots+22$

3.2.1 Working Principle of PLL Synthesizer ……............. 23

3.2.2 Phase Noise Analysis …................................. 25

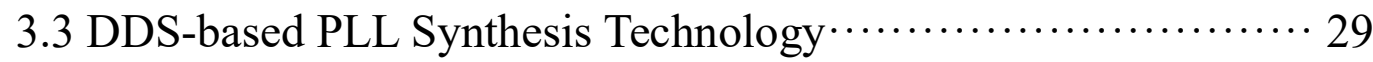

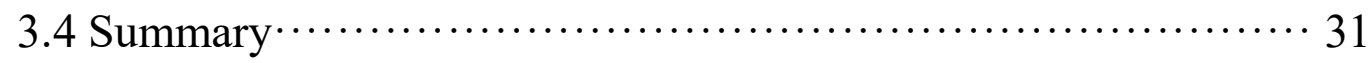

4 FMCW Radar System Design and Test 32

4.1 Radar System Design …...................................... 33

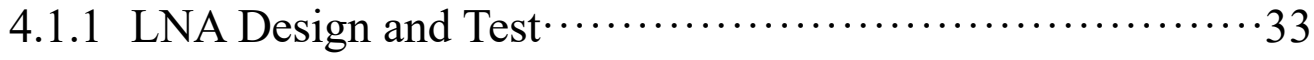

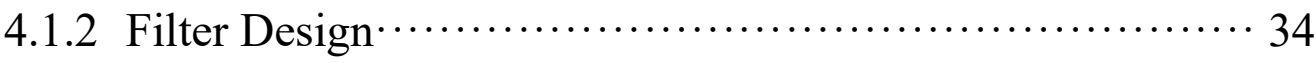

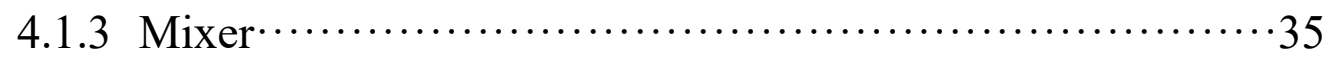




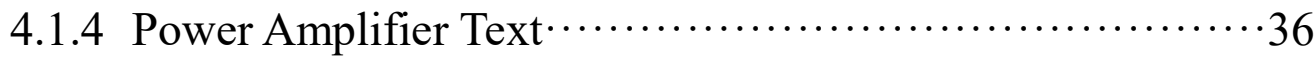

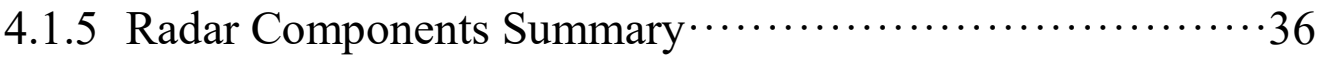

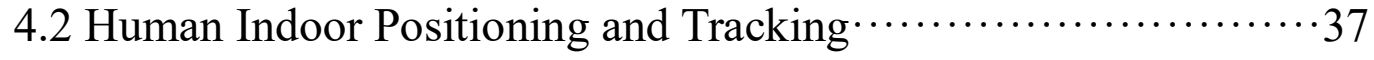

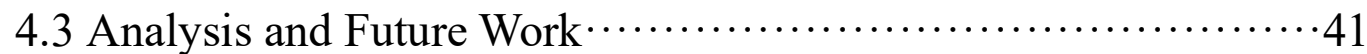

$\begin{array}{ll}\text { Appendix } & 48\end{array}$

Bibliography 


\section{Chapter 1}

\section{Introduction}

The word "radar" is an acronym derived from the phrase RAdio Detection And Ranging and it is applied for detecting and tracking objects at certain distances. The basic principle of radar is simple - signals traveling at the speed of light are transmitted, reflected off a target and then returned as an echo.

\subsection{Research Background}

The history of radar dated back to experiments by Heinrich Hertz in 1886 that showed that radio waves were reflected by metallic objects, and in 1900 Tesla described a concept for electromagnetic detection and velocity measurement in an interview, [1]. In 1903 and 1904 , the German engineer Hülsmeyer experimented with ship detection by radio wave reflection, an idea advocated again by Marconi in 1922. In that same year, Taylor and Young of the U.S. Naval Research Laboratory (NRL) demonstrated ship detection by radar and in 1930 Hyland, also of NRL, first detected aircraft (albeit accidentally) by radar, setting off a more substantial investigation that led to a U.S. patent for what would now be called a continuous wave (CW) radar in 1934.

Early radar development was driven by military necessity, including surveillance, navigation, and weapons guidance for ground, sea, air, and space vehicles. And now, radar enjoys an increasing range of applications, [2]. One of the most common is the police traffic radar used for enforcing speed limits. Another is the "weather radar" familiar to every viewer of local television news. Another radar application is found in the air traffic control systems used to guide commercial aircraft. Finally, satellite and airborne radar is an important tool in mapping earth topology and environmental characteristics such as water and ice conditions, forestry conditions, land usage. While 
this sketch of radar applications is far from exhaustive, it does indicate the breadth of applications of this remarkable technology.

Radar can be classified as primary radar and secondary radar, which is shown in figure 1.1: a primary radar transmits signals with high frequency toward the targets. The transmitted signals are reflected by the target and then received by the same radar. And secondary radar works with active answer signals.

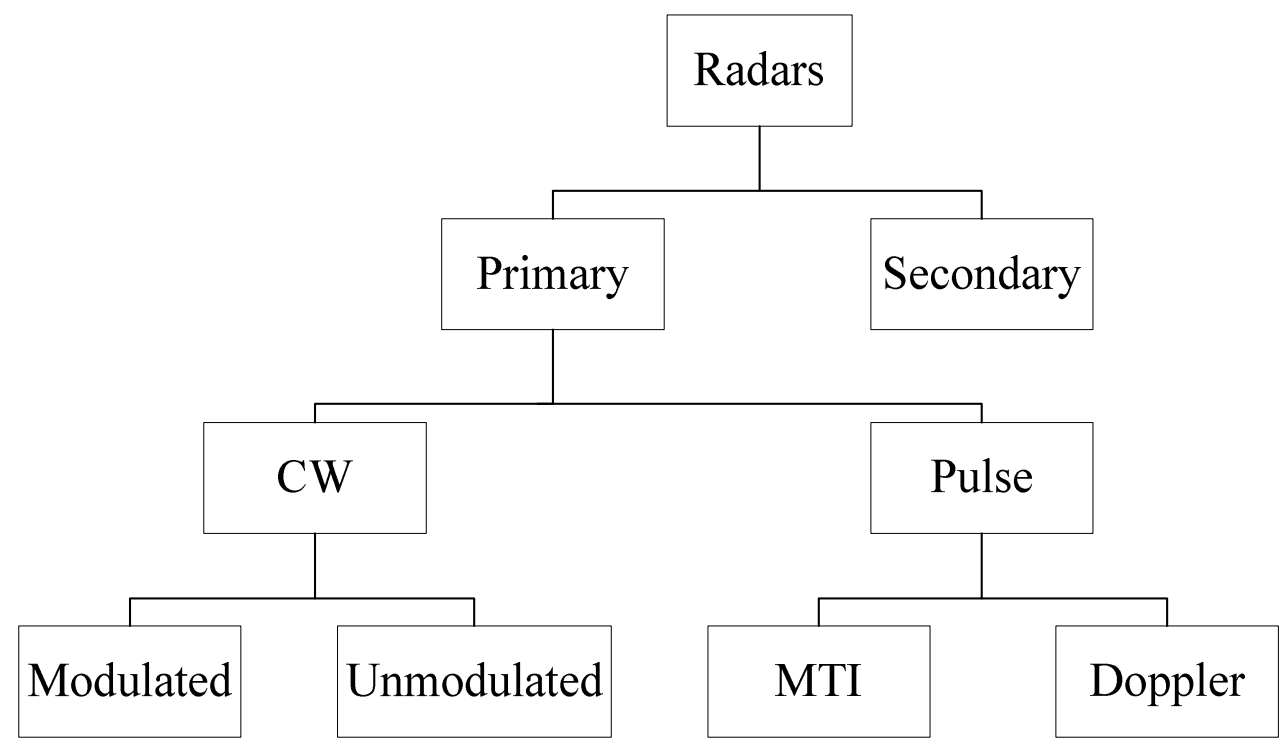

Figure 1.1 Simplified classification of radar

CW radars continuously transmit a high-frequency signal and the reflected signal is also received and processed continuously. The transmitted signal of unmodulated radar is constant in amplitude and frequency. Transmitting unmodulated power of CW radar can only measure the speed using the Doppler effect, but it cannot measure a range and cannot differ between two reflecting objects. This is achieved in modulated CW radars using the frequency shifting method. In this method, a signal changing in frequency constantly around a fixed reference is used to detect objects. Pulsed radar transmits high power, high-frequency pulses toward the target, then it waits for the echo of the transmitted signal before it transmits a new pulse.

Each method has its advantages over the other. The advantages of pulsed radar include increased range, lower power consumption, and it does not rely on the Doppler Effect to determine movement. Continuous wave radar benefits include a continuous updating of target, higher resolution, and doesn't have the minimum target distance. 


\subsection{Research Status of FMCW radar}

As the name suggests, frequency modulated continuous wave (FMCW) radar is a technique for obtaining range information from a radar by frequency modulating a continuous signal. The technique has a very long history, but in the past its use has been limited to certain applications, such as radio altimeters. However, there is now renewed interest in the technique for three main reasons, [3]. First, the most general advantage possessed by FMCW radar is that the modulation is readily compatible with a wide variety of solid-state transmitters. Second, the frequency measurement which must be performed to obtain range measurement from such a system can now be performed digitally, for example, using a processor based on the fast Fourier transform (FFT).

Benefits of FMCW over other modulated $\mathrm{CW}$ waveforms are: 1) the ability to control the range and to obtain very efficient use of the spectrum; 2) the ease with which the range resolution can be changed, and the way in which very high range resolutions can be obtained without requiring wide IF and video bandwidths.

During the second world war, FMCW radar was used for bomb aiming radars and in 1946 an FMCW surveillance radar was built. This obtained its modulation by "pushing" a magnetron. It did not work very well, and the reason for building it is unclear.

Bamck [4] first discussed the application of moving target indication (MTI) type processing to FMCW radar. In the meantime, Fuller may have been the first to apply FMCW to automotive radar, but in this case the modulation was almost a "side effect" of the use of frequency scanning to scan the beam.

A major step forward was made in the mid 1970's when digital signal processing became available to perform the signal processing to extract the range information from the received signals. Before digital processing became available, many earlier FMCW radars, such as those discussed above, used single band-pass filters to detect signals at a single range, and varied the sweep rate systematically to search all the possible range gates, [5]. This makes very inefficient use of the radar's observation times. On the other hand, FMCW radio altimeters have long used a closed-loop system to adjust the sweep period to keep the beat frequency from the ground within a single range bin. Since only a single range-gate is required, this approach achieves efficient use of the energy without needing to process many range gates. Modern radio altimeters, however, do 
also use digital (FFT-based) processing as well as this technique. It is now commonplace to use a FFT, or other digital frequency analysis techniques, to analyze the mixture of beat frequencies corresponding to the targets at different ranges.

In 1998, McClanahan and other researchers in United States developed a ranging system based on pulsed radars, of which the center frequency was $3.2 \mathrm{GHz}$, the error of test data was less than 10\%. In 1999, Li developed a FMCW radar obtaining range and angle information of obstacles. Its working frequency was $77 \mathrm{GHz}$, transmitting signal linearity was better than $0.5 \%$, maximum range was $100 \mathrm{~m}$, range resolution was less than $1 \mathrm{~m}$. It had a wide application for vehicle collision avoidance system because of the simple structure.

In 1999, William H. Haydl developed a FMCW radar integrated on a $0.88 \mathrm{~mm}^{2}$ single chip. Its working frequency is $94 \mathrm{GHz}, \mathrm{DC}$ input power is $0.7 \mathrm{~W}$, RF power is $10 \mathrm{~mW}$. The receiver had $6 \mathrm{~dB}$ noise figure and $10 \mathrm{~dB}$ gain. The whole front end included VCO, PA, LNA, mixer and coupler. In 2003, Musch used N-fractional PLL to develop a FMCW radar. The system has high linearity and measurement error was about $0.8 \mathrm{~mm},[6]$.

In 2016, Gitae Pyo [7] and his team developed a CMOS transceiver IC for a singleantenna FMCW radar at $\mathrm{K}$ band. The transceiver achieved the output power of -1.6 $\mathrm{dBm}$, phase noise of $-105 \mathrm{dBc} / \mathrm{Hz}$ at $1 \mathrm{MHz}$ offset, receiver gain of $15.3 \mathrm{~dB}$, and the noise figure of $11.6 \mathrm{~dB}$. including a $1.7 \mathrm{~mm} \times 0.9 \mathrm{~mm}$ pad.

Nowadays, FMCW radar is widely used in various fields from civil to military applications. It can be used for imaging purposes. Automotive FMCW radar can be used to record velocity violations on roads. It can also be used as driver intelligence systems to improve driving conditions and avoid collisions. It can be also employed for ship navigation and identification. FMCW radars can also be used target detection under the ground clutter environment, [8]. Some authors presented FMCW radars for geosciences to measure wind speed and directions.

On the other hand, it is frequently used for indoor human tracking for indoor healthcare scenarios, such as fall detection of elderly people or breath detection, and intelligent housing system. FMCW radar for indoor human tracking is becoming more portable, more precise and cheaper. In this work, a FMCW radar system at $2.4 \mathrm{GHz}$ is proposed for intelligent housing systems. 


\subsection{Research Status of PLL Frequency Synthesizer}

The phase locked loop is a control system that can generate an output signal whose phase is related to the phase of an input signal. While there are several differing types, it is easy to initially visualize as an electronic circuit consisting of a variable frequency oscillator and a phase detector. The oscillator generates a periodic signal, and the phase detector compares the phase of that signal with the phase of the reference signal, adjusting the oscillator to keep the phases matched, [9]. Bringing the output signal back toward the input signal for comparison is called a feedback loop since the output is "fed back" toward the input forming a loop.

PLLs date back to the 1920 s, but their popularity and applications took off with the introduction of the monolithic PLL, [10]. The 4046 CMOS Micropower PLL, which RCA introduced in the 1970s, is one of the early PLL ICs. These ICs found use in many applications, including frequency synthesis, FM demodulation and modulation, voltage-to-frequency conversion, and data synchronization. The 4046 integrated two types of phase detectors - a linear mixer and an edge-triggered phase/frequency detector - with a VCO (voltage-controlled oscillator) and an output buffer that allowed designers to use the tuning voltage for demodulation applications.

The most common type of PLL for SOC (system-on-chip) applications is the frequency-multiplying PLL. This type of PLL generates a high-frequency clock from a low-frequency crystal or another reference. Applications for frequency-multiplying PLL are widespread and include logic clocking and RF local-oscillator synthesis. Figure 1.2 shows the history and development of radar and PLL. 


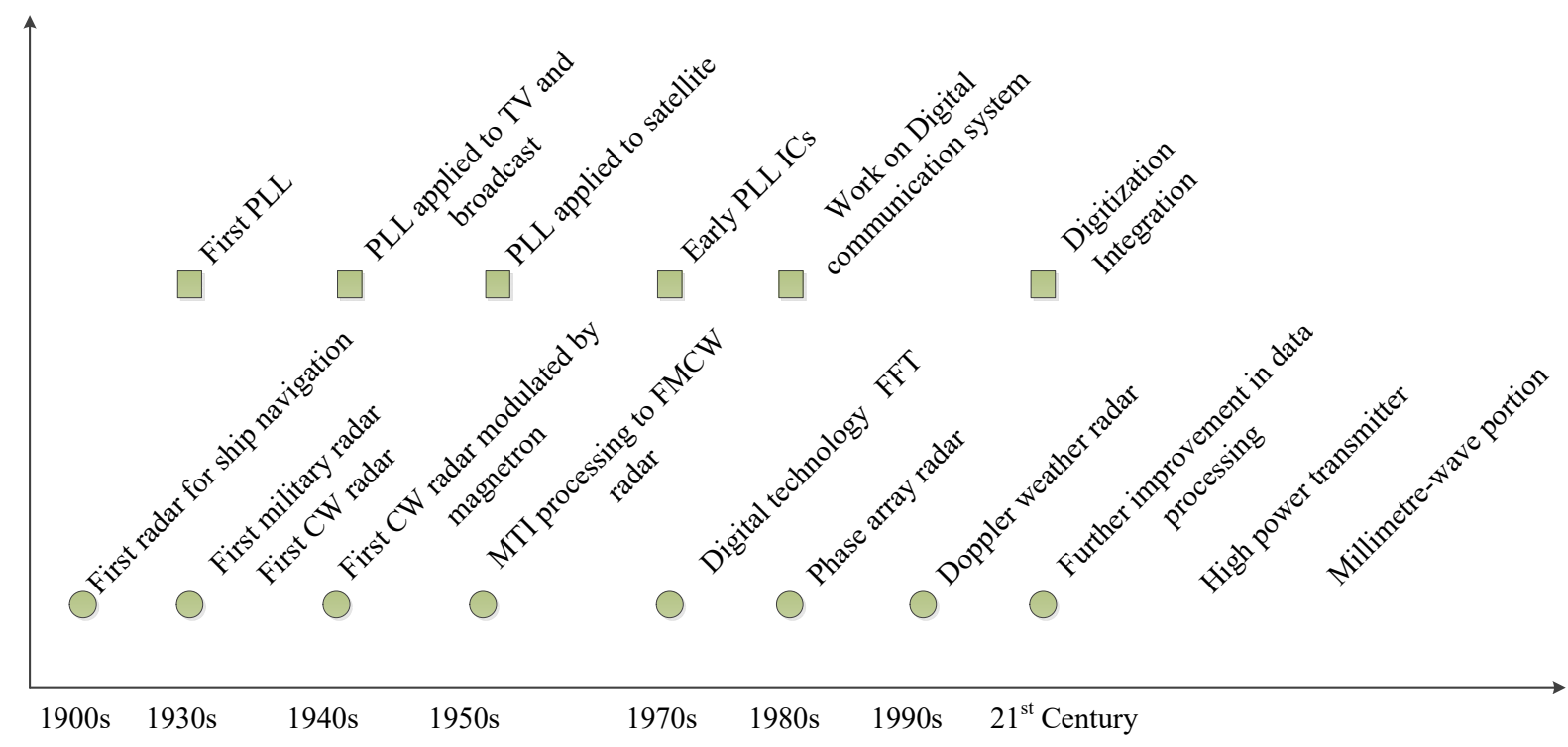

Figure 1.2 History of the development of radar and PLL

\subsection{Thesis Outline}

This thesis proposes a FMCW radar at ISM band for indoor human positioning and tracking, including research about DDS-based PLL frequency synthesizer and homodyne receiver architecture. The content of this thesis is organized as follows:

In Chapter 2, the thesis presents the working principle and equations of FMCW radar, and parameters of the indoor FMCW radar, including range and velocity information, are given. Then in Microwave Office, the whole radar system is simulated.

In Chapter 3, the thesis designs the frequency synthesizer for the FMCW radar. The PLL frequency synthesizer is based on a DDS board. In this chapter, the thesis discusses the working principle of DDS and PLL, and gives the measurement and analysis of power spectrum and phase noise of the DDS-based PLL board.

In Chapter 4, the thesis discusses the design of FMCW radar transmitter and homodyne receiver. Test and analysis of each component is given. And the thesis does test and analysis of the whole FMCW radar system. It shows that the whole transceiver has a good performance to position and track objects in 10 meter with $2 \%$ error. 


\section{Chapter 2}

\section{FMCW Radar Theory}

In the past decade, portable radar systems have experienced tremendous growth. Such rapid growth has created demand for portable wireless devices that are smaller, lighter, cheaper and of higher performance than ever, and this drives IC designers and engineers to innovate new system architectures and circuit topologies. In this work, we choose frequency modulated continuous wave (FMCW) radar to implement indoor positioning. FMCW radar, it is a type of radar system where a stable frequency continuous-wave radio energy is transmitted and then received from reflecting objects. This work adopts a homodyne architecture in FMCW radar receiver.

\subsection{Working Principle of FMCW radar}

As described above, FMCW radar is a special type of radar sensor which radiates continuous transmission power like a simple continuous wave radar. In contrast to normal $\mathrm{CW}$ radar, FMCW radar can change its operating frequency during the measurement: that is, the transmission signal is modulated in frequency (or in phase).

Simple continuous wave radar devices without frequency modulation have the disadvantages [11] that they cannot determine target range because they lack the timing mark necessary to allow the system to time accurately the transmit and receive cycle and to convert this into range. Such a time reference for measuring the distances of stationary objects, but can be generated using frequency modulation of the transmitted signal. In this method, a signal is transmitted, which increases or decreases in the frequency periodically. When an echo signal is received, that change of frequency gets a delay $\Delta \mathrm{t}$ similar to the pulse radar technique. In pulse radar, however, the runtime must be measured directly. For a FMCW radar, the distance measurement is accomplished by comparing the frequency of the received signal to a reference, which is usually the transmission signal. And the duration of the transmission signal is 
substantially greater than the required receiving time for the installed distance measuring range.

There are several modulation patterns used for different measurement purposes: sawtooth modulation, triangular modulation, square-wave modulation (simple frequency-shift keying, FSK) and stepped modulation (staircase voltage). Sawtooth modulation is used in a large range combined with a negligible influence of Doppler frequency. And for square-wave modulation, it is used for a very precise distance measurement at close range by phase comparison of the two echo signal frequencies. But the disadvantage is that the echo signals from several targets cannot be separated from each other, and that this process enables only a small unambiguous measuring range.

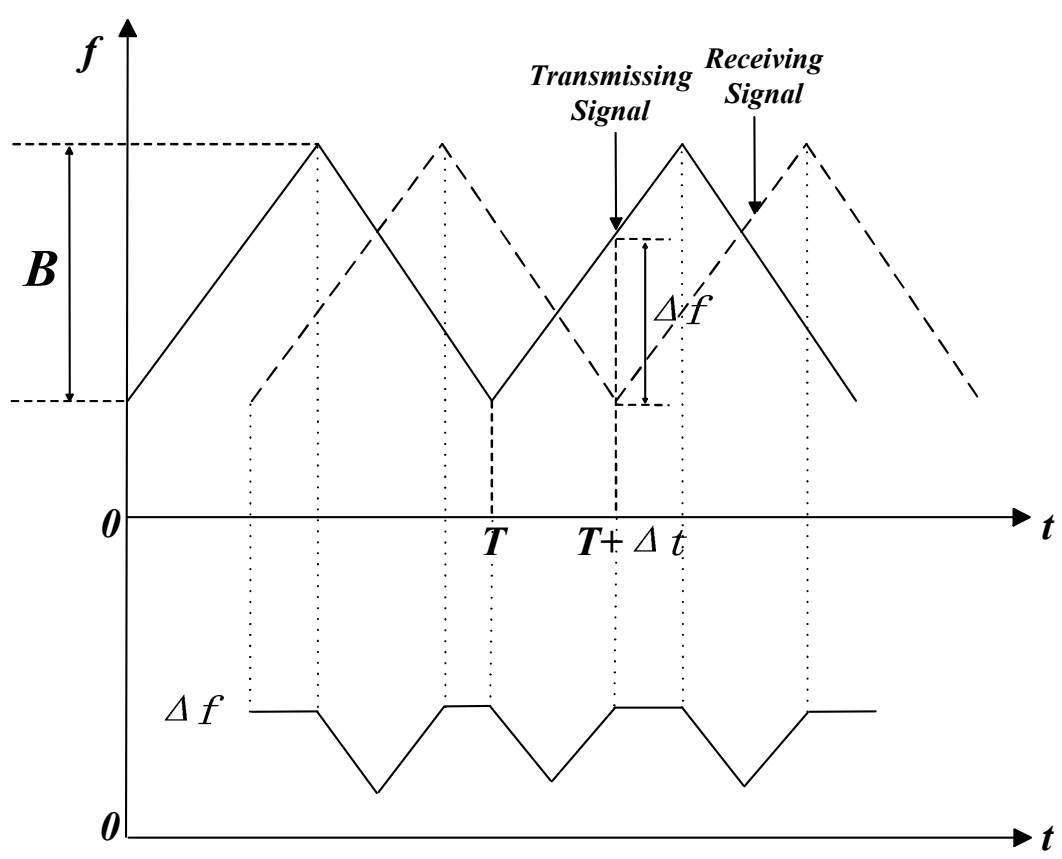

Figure 2.1 Working principle of FMCW radar for stationary targets

In this thesis, triangular modulation is issued, the carrier signal of the radar is frequency-modulated by triangular waves. The radar transmits and receives signals with a frequency deviation, as shown in figure 2.1. The frequency difference is proportional to the time difference between the transmitted and received signals, which in turn is proportional to the distance between the transmitter and target.

Assuming that the transmitter frequency increases linearly with time and that there is a reflecting object at a distance, the time varing amplitude, frequency and phase is shown below: 


$$
\begin{gathered}
u_{S}=A_{s} \cos \varphi_{S}(t) \\
f_{S}(t)=f_{c}+\frac{2 B}{T} t \\
\phi_{S}(t)=2 \pi \int_{0}^{t} f_{S}(t) d t+\varphi_{s o}=2 \pi\left(f_{c} t+\frac{B}{T} t^{2}\right)+\varphi_{s o}
\end{gathered}
$$

where $A_{S}$ is the signal amplitude, $f_{c}$ is the carrier frequency, $\varphi_{s o}$ is the initial phase. $B$ is the sweep rate in $\mathrm{Hz}$. An echo signal will return after the transit time $\Delta \mathrm{t}$, and the phase of the received signal can be expressed as:

$$
\varphi_{E}(t)=\varphi_{S}(t-\Delta t)=2 \pi\left(f_{c}(t-\Delta t)+\frac{B}{T}(t-\Delta t)^{2}\right)+\varphi_{s o}
$$

The beat frequency component can be written as:

$$
\begin{aligned}
u_{d}(t) & =U_{D} \cos \left(\varphi_{S}(t)-\varphi_{E}(t)\right) \\
& =U_{D} \cos \left[2 \pi\left(\frac{2 B}{T} t \Delta t+f_{c} \Delta t-\frac{B}{T} \Delta t^{2}\right)\right]
\end{aligned}
$$

where $U_{D}$ is the amplitude of the beat note signal. If there is no Doppler frequency shift, the beat frequency is a measure of the target's range:

$$
\Delta t=\frac{2 r_{o}}{c_{0}}
$$

According to triangle relationship:

$$
\frac{\Delta t}{\Delta f}=\frac{T / 2}{B}
$$

From equation (2.6) and (2.7), the distance from target can be expressed as:

$$
R=\frac{c_{o} T}{4 B} \Delta f
$$




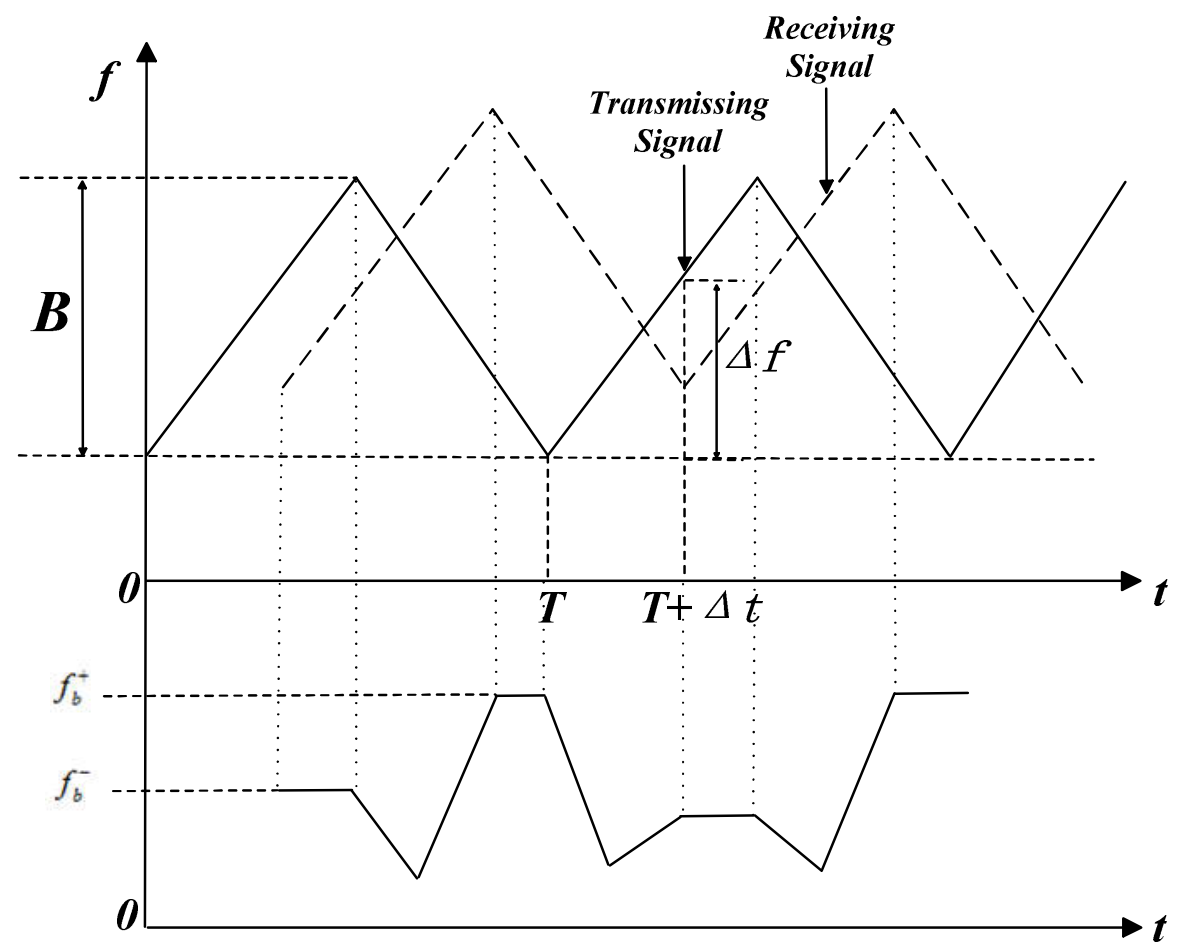

Figure 2.2 Working principle of FMCW radar for moving targets

For a moving object [13], as figure 2.2 illustrates, the frequency of received waves is shifted because of time delay and Doppler shift, which results in two different beat frequencies. Denoting the modulation range and start frequency as $B$ and $f_{o}$, the frequency of transmitting signal is shown below:

$$
\begin{gathered}
f_{t}^{+}(t)=f_{o}+k t \quad 0 \leq t<T / 2 \\
f_{t}^{-}(t)=f_{o}+2 B-k t \quad T / 2 \leq t<T
\end{gathered}
$$

of which the instantaneous phase is

$$
\begin{gathered}
\phi_{t}^{+}(t)=2 \pi \int_{0}^{t} f_{t}^{+} d t+\theta_{o}=2 \pi f_{o} t+\pi k t^{2}+\theta_{o} \quad 0 \leq t<T / 2 \\
\phi_{t}^{-}(t)=2 \pi \int_{0}^{t} f_{t}^{-} d t+\theta_{o}=2 \pi\left(f_{o}+2 B\right)_{o}(t-T / 2)-\pi k\left(t^{2}-T^{2} / 4\right)+\phi_{t}^{+}(\mathrm{T} / 2) \quad T / 2 \leq t<T
\end{gathered}
$$

Thus, the instantaneous phase of echo signal can be expressed as:

$$
\begin{array}{r}
\phi_{r}^{+}(t)=2 \pi f_{o}(t-\tau(t))+\pi k(t-\tau(t))^{2}+\theta_{o} \quad \tau \leq t<T / 2+\tau \\
\phi_{r}^{-}(t)=2 \pi\left(f_{o}+2 B\right)_{o}(t-\tau(t)-T / 2)-\pi k\left[(t-\tau(t))^{2}-T^{2} / 4\right]+\phi_{t}^{+}(\mathrm{T} / 2) \quad T / 2+\tau \leq t<T
\end{array}
$$

When $\phi_{b}^{+}=\phi_{t}^{+}-\phi_{r}^{+}$and $\phi_{b}^{-}=\phi_{t}^{-}-\phi_{r}^{-}$, the beat frequency is obtained: 


$$
\begin{gathered}
f_{b}^{+}(t)=\frac{1}{2 \pi} \frac{d \phi_{b}^{+}}{d t}=k \tau(t)-f_{d} \quad \tau \leq t<T / 2 \\
f_{b}^{-}(t)=\frac{1}{2 \pi} \frac{d \phi_{b}^{-}}{d t}=k \tau(t)+f_{d} \quad T / 2+\tau \leq t<T
\end{gathered}
$$

Since $T$ is much greater than $\tau$ :

$$
\begin{array}{ll}
f_{b}^{+}(t)=k \tau(t)-f_{d} & \tau \leq t<T / 2 \\
f_{b}^{-}(t)=k \tau(t)+f_{d} & T / 2 \leq t<T
\end{array}
$$

where $f_{d}=f_{\text {delay }}+f_{\text {doppler }}=\frac{t_{d} \times B}{T / 2}+\frac{2 f_{c} \times v_{r}}{c}, t_{d}=\frac{2 r_{o}}{c}$

As long as we can get the value of two beat frequency $f_{b}^{+}(t)$ and $f_{b}^{-}(t)$, we can get the value of distance and velocity:

$$
\begin{array}{r}
r_{o}=\frac{c_{o} T}{4 B}\left(\frac{f_{b}^{+}+f_{b}^{-}}{2}\right) \quad v_{r}=\frac{c_{o}}{2 f_{c}}\left(\frac{f_{b}^{+}-f_{b}^{-}}{2}\right) \\
r_{o}=\frac{c_{o} T}{4 B}\left(\frac{f_{b}^{+}+f_{b}^{-}}{2}\right) \quad v_{r}=\frac{c_{o}}{2 f_{c}}\left(\frac{f_{b}^{+}-f_{b}^{-}}{2}\right)
\end{array}
$$

The frequency range of ISM band is from $2.4 \mathrm{GHz}$ to $2.5 \mathrm{GHz}$, the maximum bandwidth $B$ is $100 \mathrm{MHz}$. Since this FMCW radar is used inside building, the detectable distance doesn't need to be very large. In this work, we assume $R_{\max }$ is 30 $\mathrm{m}$, it's easy to get delay time for maximum range:

$$
t_{d}=\frac{2 R_{\max }}{c_{o}}=0.2 \mu \mathrm{s}
$$

The modulation period should be larger than the delay time for the maximum range. According to papers [31]-[36], most modulation periods of FMCW radar at $2.4 \mathrm{GHz}$ are from $1 \mathrm{~ms}$ to $10 \mathrm{~ms}$. In this thesis, we use ADF4158 PLL synthesizer as frequency source. In ADF4158 chip, the minimum modulation period for $2.4-2.5 \mathrm{GHz}$ is $0.8 \mathrm{~ms}$ [14]. In this system, we set the modulation period as $1 \mathrm{~ms}$. According to equation (2.8), the maximum beat frequency can be determined by:

$$
f_{\mathrm{b} \max }=\frac{4 B R_{\max }}{c T}=40 \mathrm{kHz}
$$

According to formula for distance, we can get the formula for velocity resolution ideally as follows, 


$$
\Delta v=\frac{c_{o}}{2 f_{c}}\left(\frac{f_{b}^{+}-f_{b}^{-}}{2}\right)=\frac{c_{o}}{2 f_{c}} f_{d}=\frac{c_{o}}{2 f_{c}} \Delta f_{r}
$$

where $\Delta f_{r}$ is frequency resolution of receiver, which depends on the frequency resolution of spectrum analyzer or FFT sampling frequency.

For FFT, the spectrum computed from the sampled signal has a frequency resolution $\Delta f_{r}$. Calculate the frequency resolution with the following equation:

$$
\Delta f_{r}=\frac{1}{T_{s}}=\frac{f_{s}}{N}
$$

where $T_{s}$ is the acquistion time, $N$ is the number of samples and $f_{s}$ is the sampling frequency. Thus, frequency resolution depends on how long the acquistion time is. And it is necessary to consider the acquistion time becasue we need to calculate distance information before the object moving. We do 25 times FFT and hope the total acquistion time should be less than $0.5 \mathrm{~s}$, then frequency resolution should be larger than $50 \mathrm{~Hz}$. According to equation (2.22), the velocity resolution is larger than $3 \mathrm{~m} / \mathrm{s}$, it can be seen that FMCW radar is not a good method to measure human indoor walking velocity because of such high velocity resolution.

\subsection{Radar Transceiver Architecture}

There are three common receiver architectures: heterodyne, homodyne and imagereject, all of which have different advantages and disadvantages. When designing a RF receiver, the choice of architecture is primarily determined by criteria including complexity, cost, power dissipation and the number of external components.

In a homodyne or direct conversion receiver, the incoming RF signal is downconverted to baseband (zero frequency) in one step by mixing with an oscillator output of the same frequency. The output baseband signal is then filtered with a low-pass filter to select the desired channel. This is illustrated in the block diagram in figure 2.3.

Using a homodyne receiver architecture, the difference frequency between the transmitted signal and received signal can be easily obtained if the frequency swept transmit signal is used for the LO down conversion. Consequently, this architecture is the preferred solution. 


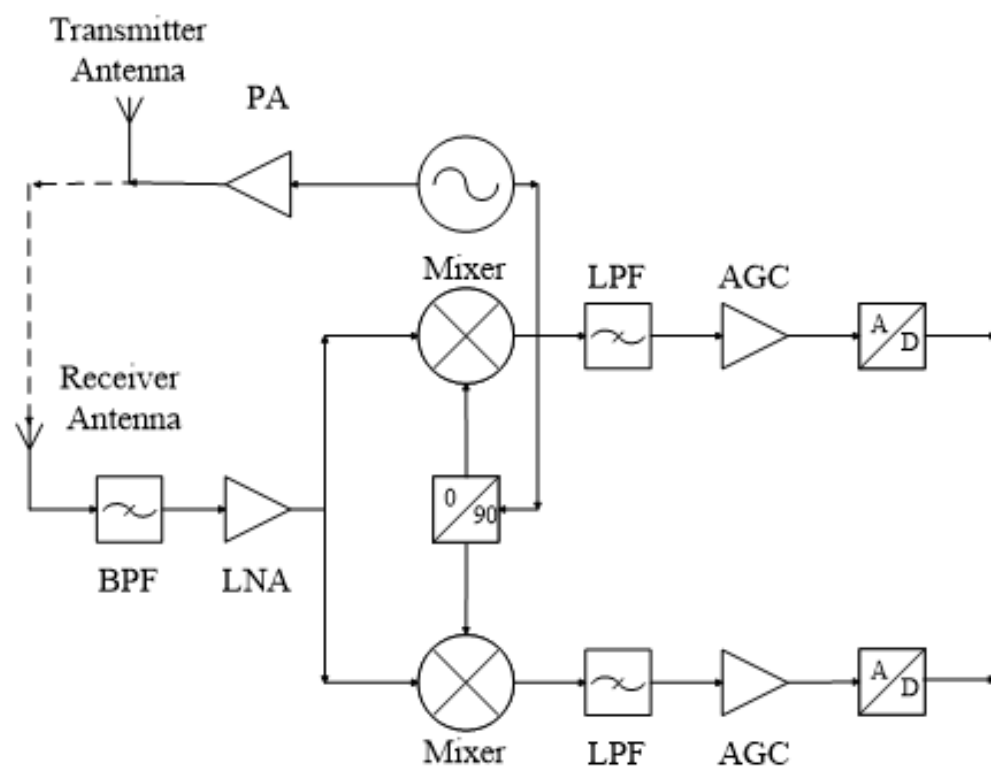

Figure 2.3 Simplified block diagram of homodyne architecture

The main advantage of a homodyne receiver [18] is that it solves the image problem because the input RF signal is down-converted directly to baseband without any IF stage. For the image problem, the homodyne receiver shown in figure 2.4 operates with double-sided signals, which overlap the positive and negative parts of the input spectrum. Consequently, the image frequency problem is circumvented because $\omega_{I F}=0$. As a result, no image filter is required.

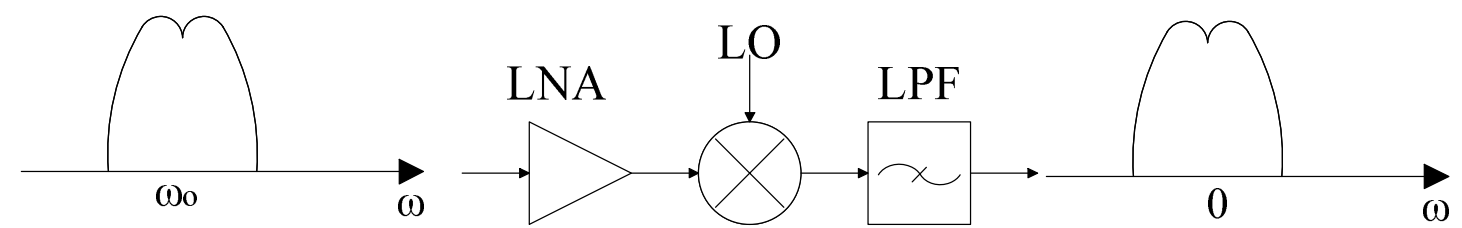

Figure 2.4 Simple homodyne receiver working principle

Another advantage is that it is easy to implement. Since it does not require any high frequency band-pass filters, which are usually implemented off-chip in a superheterodyne receiver for appropriate selectivity, the homodyne requires less number of external components. However, the homodyne architecture does suffer DC offsets. Also, since the mixer output is a baseband signal, it can easily be corrupted by the large flicker noise of the mixer, especially when the incoming RF signal is weak.

LO leakage can result in DC offset, when the isolation between the LO port and the inputs of the mixer and the LNA is not infinite. The leakage signal appearing at the inputs of the LNA and mixer is mixed with the LO signal, which provide a DC 
component at the output of the mixer. This phenomenon is called self-mixing. Another phenomenon arises if a large interferer leaks from the LNA or mixer input to the LO ports and is multiplied by itself. The principle is similar with self-mixing. And it is exacerbated if self-mixing varies with time.

Even-order nonlinearity is also a problem in homodyne down conversion systems. [19] Two strong interferers will generate a low-frequency beat. Because mixers exhibit a finite isolation from the RF input to the IF output due to asymmetry in the mixing core, the low-frequency beat will appear in the IF port. Besides, the mixer RF port may also suffer from even-order distortion, requiring special attention in the design.

Next, some basic parameters of FMCW radar system are shown below:

(1) Working Frequency: The working frequency of a transmitter and a receiver includes center frequency and bandwidth. In this thesis, the FMCW radar uses industrial, scientific and medical (ISM) radio bands, which is reserved internationally for the use of radio frequency (RF) energy for industrial, scientific and medical purposes other than telecommunications. The working frequency is from $2.4 \mathrm{GHz}$ to $2.5 \mathrm{GHz}$, so the center frequency is $2.45 \mathrm{GHz}$ and bandwidth is $100 \mathrm{MHz}$.

(2) Output Power: Output power is the power sent to the antenna from the last stage of the transmitter. According to the FCC rules, the transmit power limit at $2.4 \mathrm{GHz}$ is $30 \mathrm{dBm}$ ( $1 \mathrm{Watt}$ ), the antenna gain is $6 \mathrm{dBi}$.

(3) Noise Figure: Noise figure is the measure of degradation of the signal-to-noise ratio (SNR), caused by components in a transceiver chain. It is defined as $N F=\frac{S N R_{\text {in }}}{S N R_{\text {out }}}$, where $S N R_{\text {in }}$ and $S N R_{\text {out }}$ are the input and output SNR respectively. If several devices are cascaded, the total noise factor can be expressed as equation (2.28), the measurement of system noise figure will be discussed in chapter 4.

$$
F=F_{1}+\frac{F_{2}-1}{G_{1}}+\frac{F_{3}-1}{G_{1} G_{2}}+\frac{F_{4}-1}{G_{1} G_{2} G_{3}}+\cdots+\frac{F_{n}-1}{G_{1} G_{2} G_{3} \cdots G_{n-1}}
$$

(4) Receiver Sensitivity: Sensitivity in a receiver is normally taken as the minimum input signal required to produce a specified output signal having a specified SNR and is defined as the minimum signal-to-noise ratio times the mean noise power. For a signal impinging on the antenna, sensitivity is known as minimum operational sensitivity (MOS): 


$$
M O S=S N R \cdot k T_{o} B \cdot N F / G
$$

where $k$ is Boltzmann's constant, $T_{o}$ is effective noise temperature of the receiver input and $G$ is system gain.

According to equation (2.21), the bandwidth of IF frequency is inversely proportional to modulation period. When $T$ increases, $B$ decreases and results in smaller receiver sensitivity. The radar equation is shown in equation (2.30). On one hand, smaller receiver sensitivity means larger detection range, and more discussion about radar equation will be shown in chapter 4 . On the other hand, we need to have smaller frequency resolution for a smaller IF bandwidth, and flicker noise at low frequency will have larger effect on power spectrum.

$$
R_{\max }=\sqrt[4]{\frac{P_{t} G_{t} G_{r} \lambda^{2} \sigma}{(4 \pi)^{3} P_{\min }}}
$$

where $P_{t}$ is transmit power, $G_{t}$ and $G_{r}$ are transmitting and receiving antenna gain, $\lambda$ is transmit wavelength, $\sigma$ is target radar cross section, $P_{\min }$ is the minimum detectable signal.

We have discussed the modeling analysis of FMCW radar, a summary of FMCW radar is shown in table 2.2:

Table 2.2 FMCW Radar Main Parameters

\begin{tabular}{ll}
\hline Radar Parameter & Value \\
\hline FMCW Waveform & Triangle \\
Frequency Deviation (Bandwidth) & $100 \mathrm{MHz}$ \\
Frequency Band & ISM Band $(2.4-2.5 \mathrm{GHz})$ \\
Maximum range & $30 \mathrm{~m}$ \\
Modulation Period & $1 \mathrm{~ms}$ \\
Maximum Beat Frequency & $40 \mathrm{kHz}$ \\
Maximum Transmitter Power & $30 \mathrm{dBm}$ \\
Band-pass Filter Range & $2.4-2.5 \mathrm{GHz}$ \\
Low-pass Filter Range & $0-40 \mathrm{kHz}$ \\
Mixer Attribute & Down Converter \\
\hline
\end{tabular}

\subsection{Summary}

After talking about the history and development of FMCW radar in chapter 1. In 
chapter 2, the thesis first discusses about working principle of FMCW radar, including range formula for stationary targets and moving targets, and then the thesis gives the model of FMCW radar for indoor positioning, including maximum range, range resolution, velocity resolution and modulation period. In section 2.2 , the thesis gives some introduction about three different types of receivers: heterodyne, homodyne and image-reject receivers. In this chapter, we choose the homodyne receiver to build the FMCW transceiver architecture. 


\section{Chapter 3}

\section{DDS-based PLL Frequency Synthesizer}

A phase locked loop is a nonlinear feedback loop control system which is used for synchronization of the frequency and phase of a locally generated signal with that of an incoming signal. The PLL includes a voltage controlled oscillator (VCO), a phase detector, serval dividers and a loop filter. On the other hand, DDS is an emerging and maturing signal generation technology, which consists of phase accumulator, lookup table and DAC converter. [20] In this thesis, we use a DDS-based PLL synthesizer as frequency source of FMCW radar transceiver. It offers very many advantages, including high levels of stability and accuracy. It is also easy to be controlled from digital circuitry such as microprocessors.

\subsection{Working Principle of Direct Digital Synthesizer}

As the name suggests this form of synthesis generates the waveform directly using digital techniques. This is different from the more familiar indirect synthesizers that use a phase locked loop as the basis of their operation.

A direct digital synthesizer operates by storing the points of a waveform in digital format, and then recalling them to generate the waveform. The rate at which the synthesizer completes one waveform then governs the frequency. The overall block diagram is shown below, but before looking at the detailed operation of the synthesizer it is necessary to look at the basic concept behind the system.

The operation can be envisaged more easily by looking at the way that phase progresses over the course of one cycle of the waveform, [21]. This can be envisaged as the phase progressing around a circle. As the phase advances around the circle, this corresponds to advances in the waveform. 


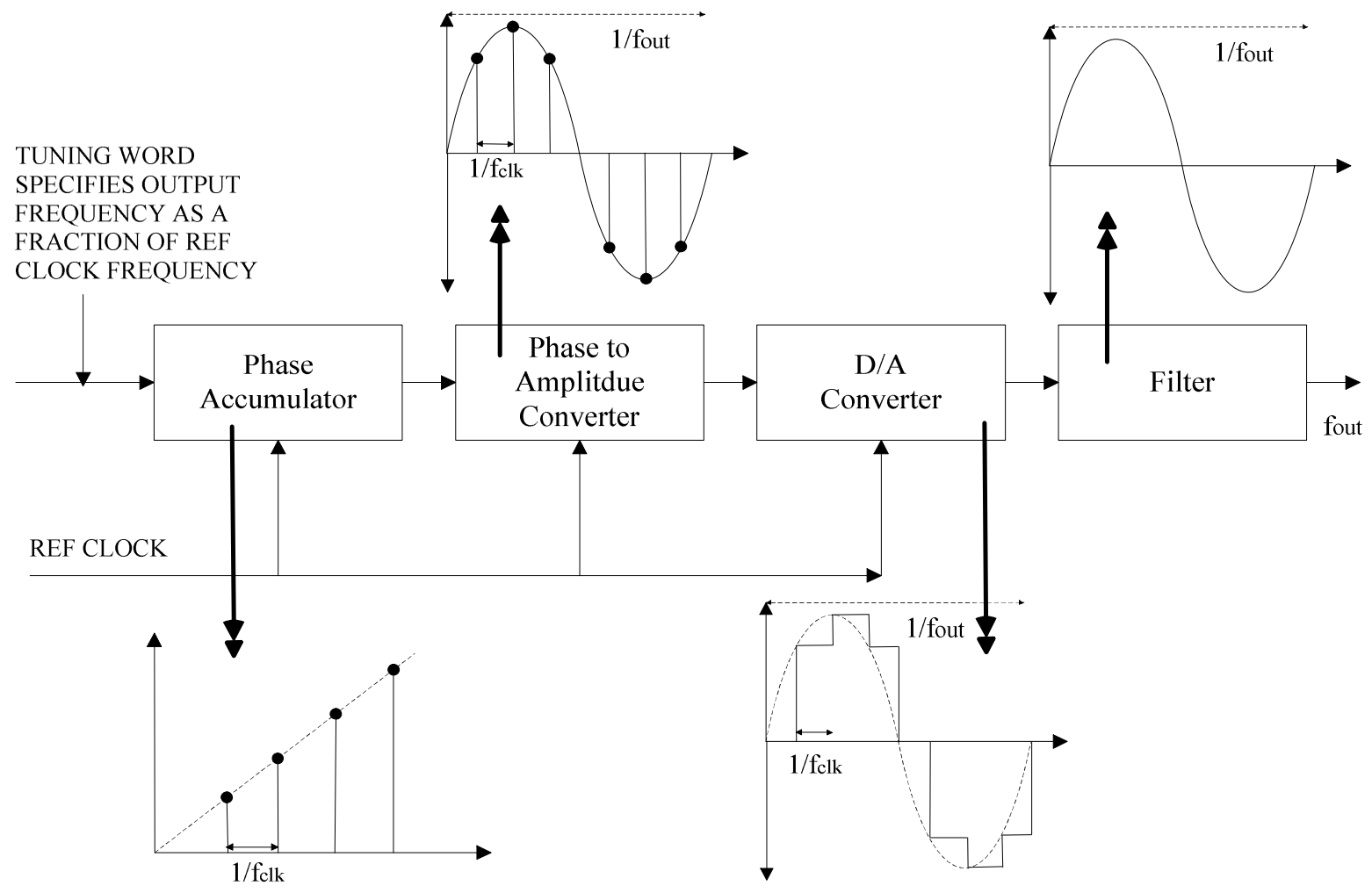

Figure 3.1 Diagram of the direct digital synthesizer, and its signal flow

The synthesizer operates by storing various points in the waveform in digital form and then recalling them to generate the waveform. Its operation can be explained in more detail by considering the phase advances around a circle as shown in Figure 3.2. As the phase advances around the circle this corresponds to advances in the waveform, i.e. the greater the number corresponding to the phase, the greater the point is along the waveform. By successively advancing the number corresponding to the phase it is possible to move further along the waveform cycle.

The digital number representing the phase is held in the phase accumulator. The number held here corresponds to the phase of the FM signal and is increased at regular intervals, [21]. In this way, it can be seen that the phase accumulator is basically a form of counter. When it is clocked, it adds a preset number to the one already held. When it fills up, it resets and starts counting from zero again. In other words, this corresponds to reaching one complete circle on the phase diagram and restarting again. 


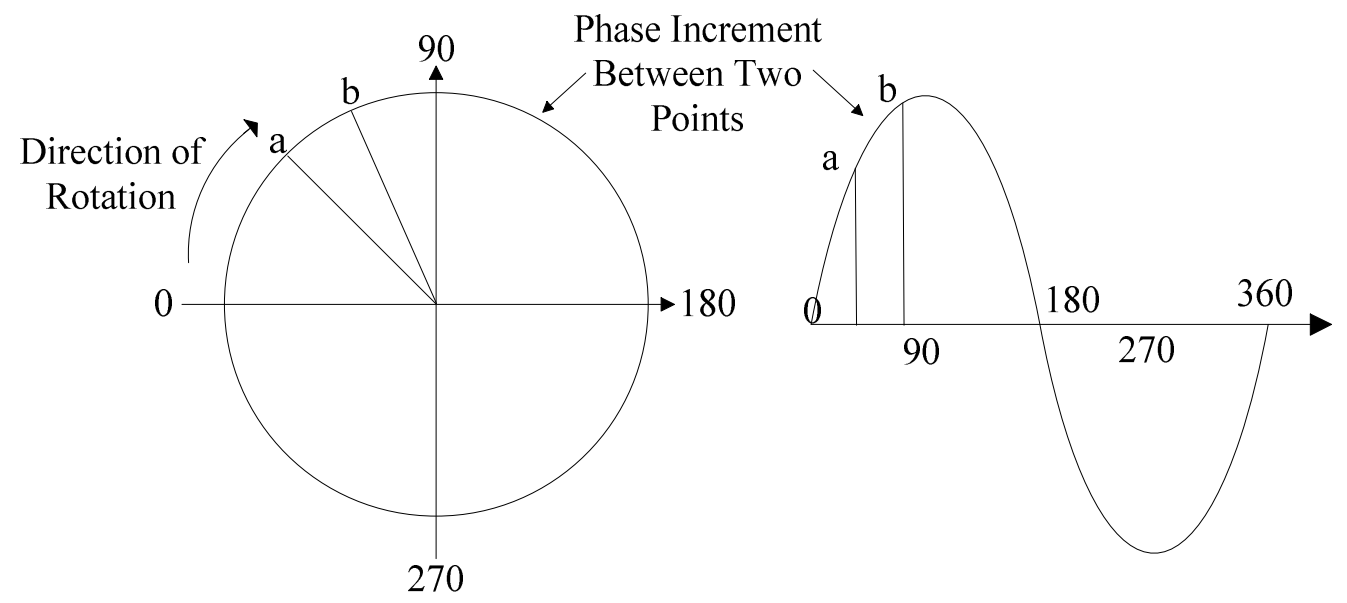

Figure 3.2 Operation of the phase accumulator in a direct digital synthesizer

Once the phase has been determined it is necessary to convert this into a digital representation of the waveform. This is accomplished using a waveform map. This is a memory which stores a number corresponding to the voltage required for each value of phase on the waveform. In the case of a synthesizer of this nature it is a sine look up table as a sine wave is required. In most cases the memory is either a read only memory (ROM) or programmable read only memory (PROM), [22]. This contains a vast number of points on the waveform, very many more than are accessed each cycle.

The next stage in the process is to convert the digital numbers coming from the sine look up table into an analogue voltage. This is achieved using a digital to analogue converter (DAC). This signal is filtered to remove any unwanted signals and amplified to give the required level as necessary.

Tuning is accomplished by increasing or decreasing the size of the step or phase increment between sample points. A larger increment at each update to the phase accumulator will mean that the phase reaches the full cycle value faster and the frequency is correspondingly high. Smaller increments to the phase accumulator value means that it takes longer to increase the full cycle value and a correspondingly low value of frequency. In this way, it is possible to control the frequency. It can also be seen that frequency changes can be made instantly by simply changing the increment value. There is no need to a settling time as in the case of phase locked loop based synthesizer.

These synthesizers do have some disadvantages, [23]. There are numbers of spurious signals which are generated by a direct digital synthesizer. The most important of these is one called an alias signal. Here images of the signal are generated on either 
side of the clock frequency and its multiples. For example if the required signal had a frequency of $5 \mathrm{MHz}$ and the clock was at $25 \mathrm{MHz}$ then alias signals would appear at 20 $\mathrm{MHz}$ and $30 \mathrm{MHz}$ as well as $45 \mathrm{MHz}$ and $55 \mathrm{MHz}$, etc.. These can be removed by a low pass filter. Also, some low level spurious signals are produced close in to the required signal. These are normally acceptable in level, although for some applications they can cause problems. Figure 3.3 shows alias signals of AD9833 DDS chip at $5 \mathrm{MHz}$ output.

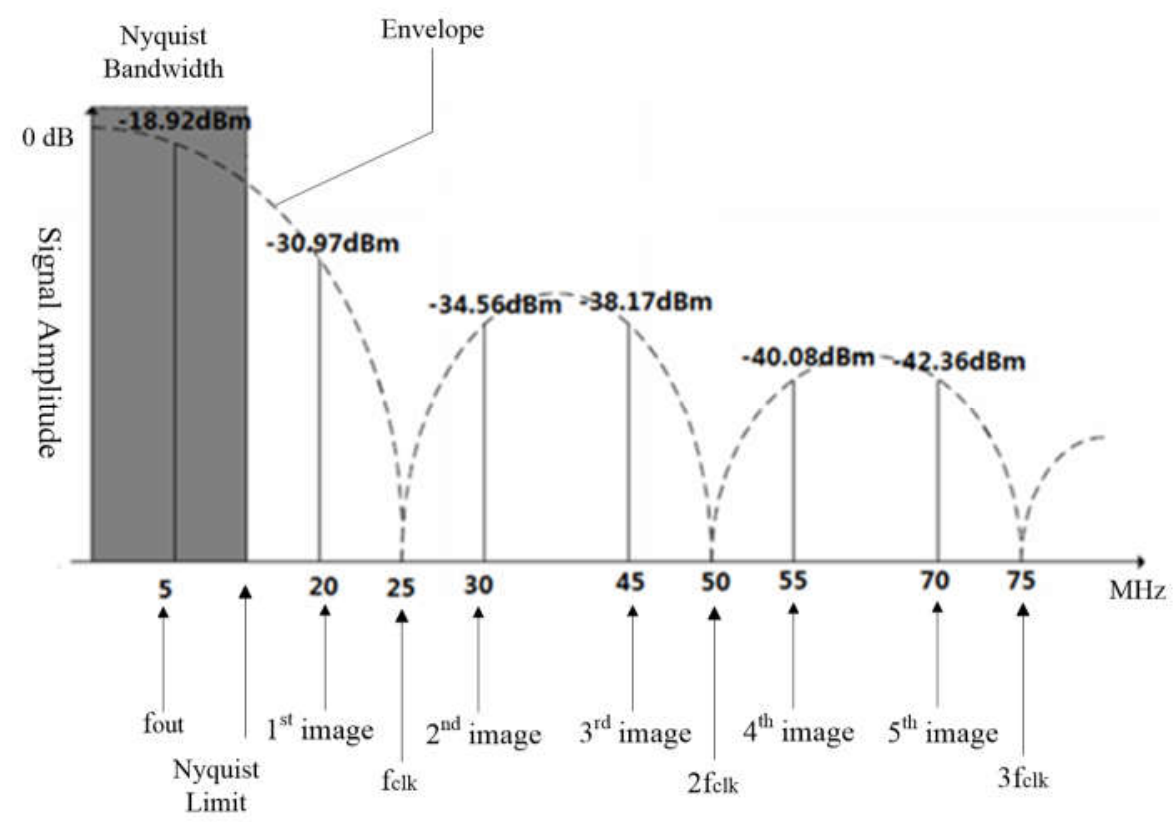

Figure 3.3 Aliasing in a DDS power spectrum

There are other "spurious" signals. Unlike a PLL-based system, the higher-order harmonics of the fundamental output frequency in a DDS system will fold back into the baseband because of aliasing. These harmonics cannot be removed by the antialiasing filter. For instance, if the clock frequency is $100 \mathrm{MHz}$, and the output frequency is 30 $\mathrm{MHz}$, the second harmonic of the $30 \mathrm{MHz}$ output signal appears at $60 \mathrm{MHz}$, but also at $100-60=40 \mathrm{MHz}$ (the aliased component). Similarly, the third harmonic (90 MHz) appears in band at $100-90=10 \mathrm{MHz}$, and the fourth at $120-100 \mathrm{MHz}=20 \mathrm{MHz}$. Higher order harmonics also fall within the Nyquist bandwidth.

Another source of spurs [25] is switching transients that arise within the internal physical architecture of the DAC. Non-symmetrical rising and falling switching characteristics such as unequal rise and fall time will also contribute to harmonic distortion. The amount of distortion is determined by the effective ac or dynamic transfer function. Transients can cause ringing on the rising and/or falling edges of the 
DAC output waveform. Ringing tends to occur at the natural resonant frequency of the circuit involved and may show up as spurs in the output spectrum.

In this thesis, we use the AD9833 board to generate reference frequency of PLL. This board is a low power, programmable waveform generator. The output frequency and phase are software programmable, allowing easy tuning. No external components are needed. The frequency registers are 28 bits wide. With a $25 \mathrm{MHz}$ clock rate, resolution of $0.1 \mathrm{~Hz}$ can be achieved. Figure 3.4 shows power spectra at different output, it can be seen that alias signals and spurs decrease with the increase of output frequency. In the DDS-based PLL frequency synthesizer, shown in figure 3.4(b), we choose 12.5 $\mathrm{MHz}$ (Nyquist Limit) as DDS output frequency.

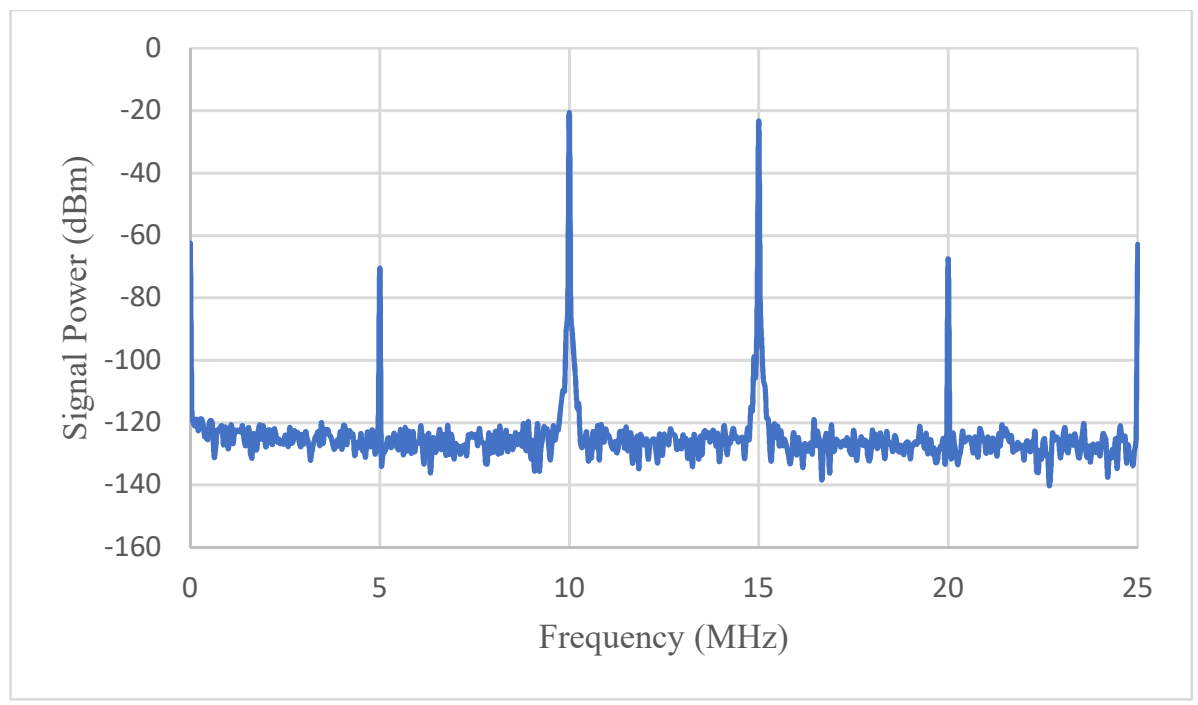

(a) $f_{\text {out }}=10 \mathrm{MHz}$

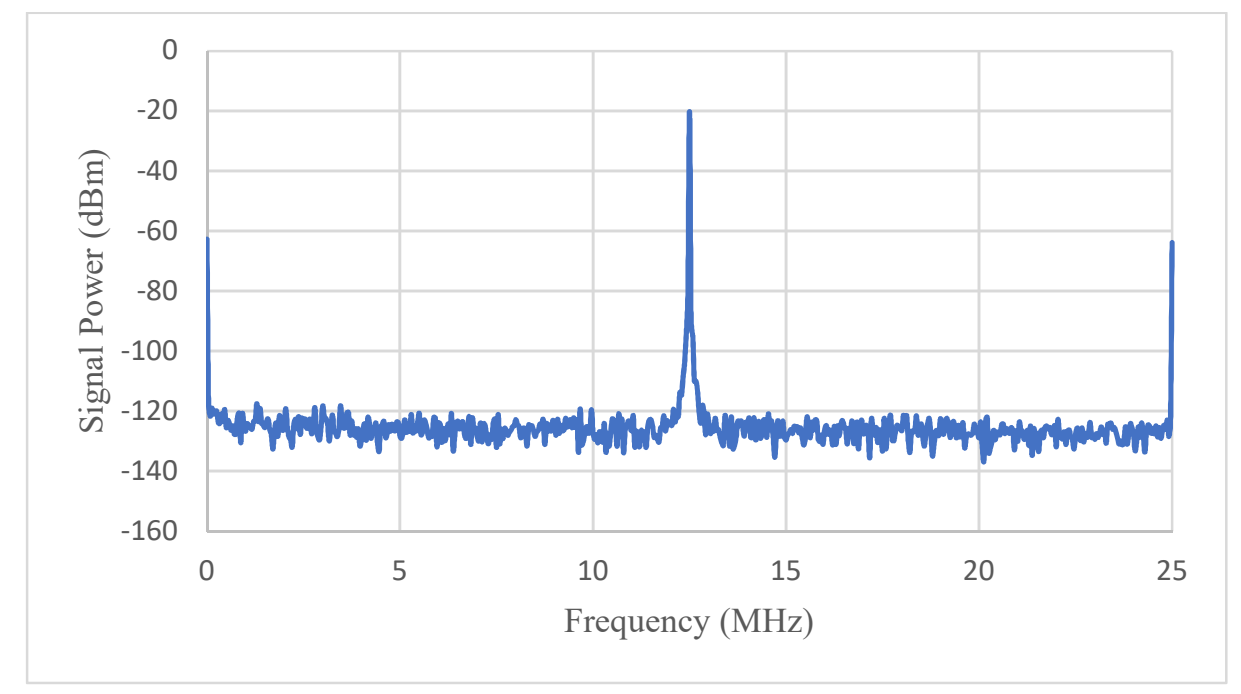

(b) $f_{\text {out }}=12.5 \mathrm{MHz}$

Figure 3.4 DDS power spectrum 
Since we use DDS signal as the reference frequency of PLL, quality of DDS power spectrum can influence output frequency of PLL. Figure 3.5 shows the PLL output power spectrum when $f_{D D S}=10 \mathrm{MHz}$ and $f_{D D S}=12.5 \mathrm{MHz}$. It is easy to find that the smaller spurs the DDS power spectrum has, the better the PLL power spectrum is. More details about DDS-based PLL will be discussed in section 3.3.

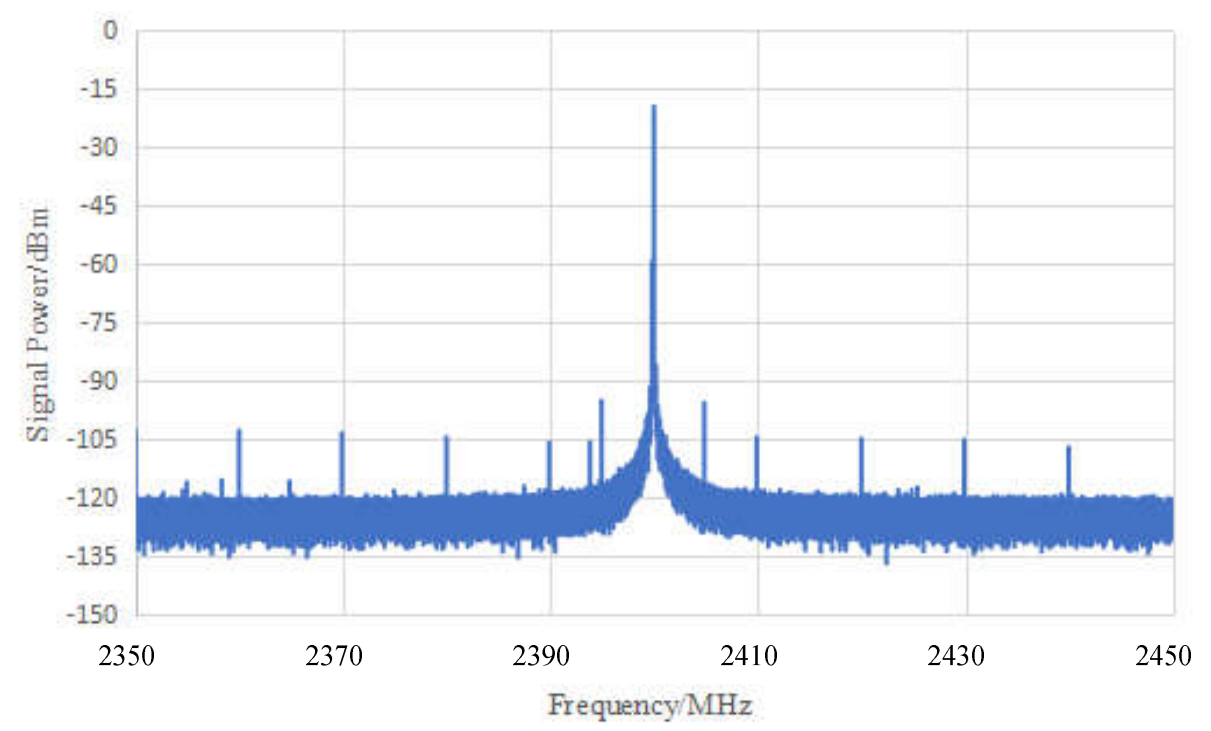

(a) PLL power spectrum when $f_{D D S}=10 \mathrm{MHz}$

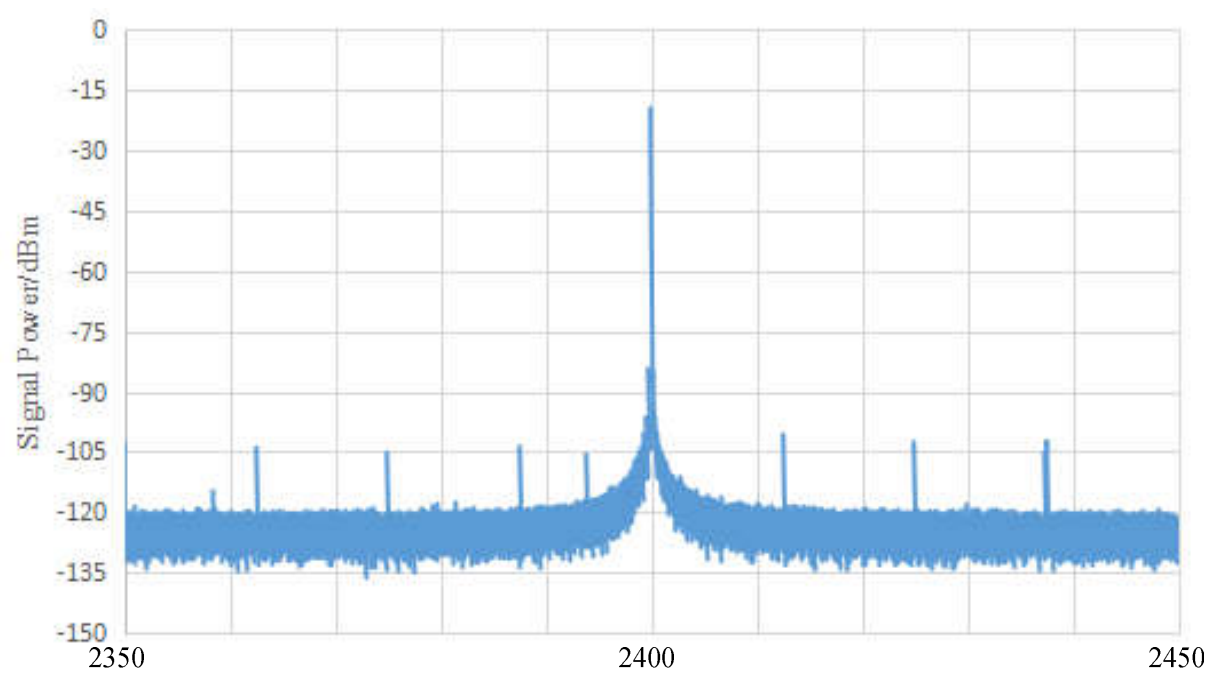

Frequency $\mathrm{MHz}$

(b) PLL power spectrum when $f_{D D S}=12.5 \mathrm{MHz}$

Figure 3.5 PLL power spectrum with different DDS signal 


\subsection{Phase-locked Loop Frequency Synthesizer}

A Phase Locked Loop (PLL) is a fundamental part of radio, wireless and telecommunication technology. It is a simple negative feedback architecture that allows economic multiplication of crystal frequencies by large variable numbers. By studying the loop components and their reaction to various noise sources, we will show that PLL is uniquely suited for generation of stable, low noise tunable RF signals for radio, timing and wireless applications.

\subsubsection{Working Principle of PLL Synthesizer}

The phase locked loop is a closed-loop control system which is used for synchronization of the frequency and phase of a locally generated signal with that of an incoming signal. It is basically a nonlinear feedback loop. The PLL consists of a voltage controlled oscillator (VCO), a phase detector, serval dividers, and a loop filter. The PLL output frequency can synchronize with reference frequency by phase tracking of PLL. The diagram of PLL is shown in figure 3.6:

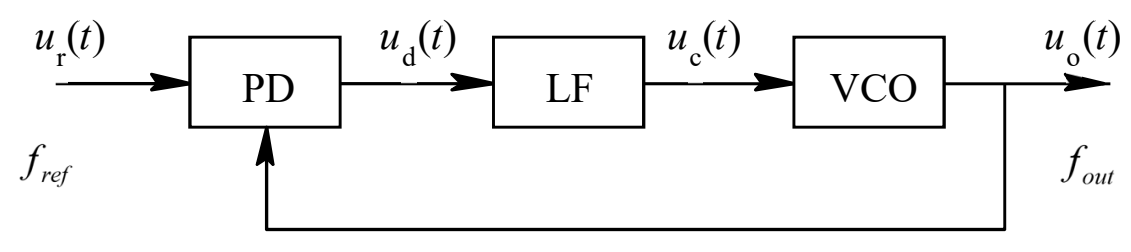

Figure 3.6 Diagram of a phase locked loop

The basic method of an integer-N phase-locked frequency synthesis is: the phase locked loop locks a high stable reference oscillator, and a programmable divider is in the loop. We change the divider ratio by programming to get stable output frequency which is $N$ times the reference frequency. Figure 3.7(a) shows the diagram of an integer-N PLL when reference frequency is $10 \mathrm{MHz}$ and output frequency is 2400.2 MHz. 


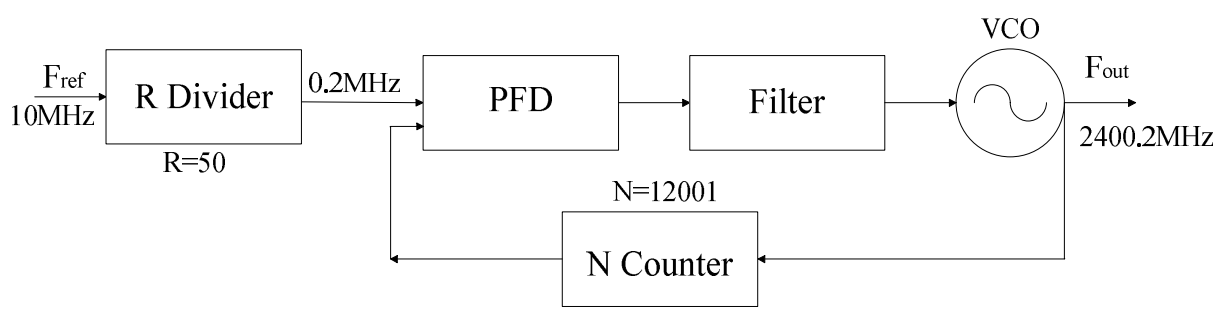

(a) Diagram of an integer-N PLL

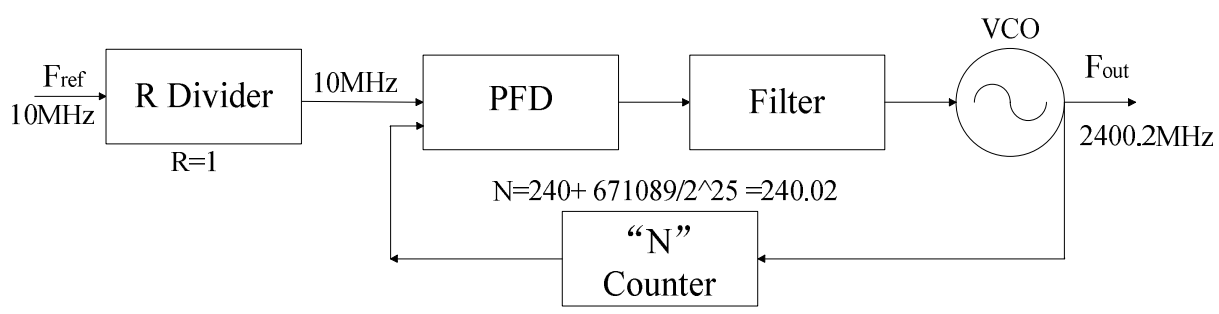

(b) Diagram of a Fractional-N PLL

Figure 3.7 Comparison between integer-N PLL and Fractional-N PLL

When the loop is locked, the output frequency is:

$$
F_{\text {out }}=\frac{N}{R} F_{r e f}
$$

An unavoidable occurrence in digital PLL synthesis is that frequency multiplication (by $N$ ), raises the signal's phase noise by $20 \log _{10}(N) \mathrm{dB}$. The phase detectors are typically the dominant source of close-in phase noise, $N$ becomes a limiting factor when determining the lowest possible phase noise performance of the output signal. We could reduce the close-in phase noise of our system by reducing the value of $N$ but unfortunately the channel spacing of an integer-N synthesizer is dependent on the value of $N$.

A phase detector is a digital circuit that generates high levels of transient noise at its frequency of operation, $F_{r}$. This noise is superimposed on the control voltage to the VCO and modulates the VCO RF output accordingly. This interference can be viewed as spurious signals at offsets of $+/-F_{r}$ (and its harmonics) around $F_{v c o}$. To prevent this unwanted spurious noise, a filter at the output of the charge pumps (called the loop filter) must be present and appropriately narrow in bandwidth. Unfortunately, as the loop filter bandwidth decreases, the time required for the synthesizer to switch between channels increases.

If $N$ could be made much smaller, $F_{r}$ would increase and the loop filter bandwidth 
required to attenuate the reference spurs could be made large enough so that it does not impact the required switching speed of our system. Once again, however, the upper limit of $F_{r}$ is bound by our channel spacing requirements. This illustrates how our desires to optimize both switching speed and spur suppression directly conflict with each other.

Fractional PLL technology has made it possible to alter the relationship between $N, F_{r}$, and the channel spacing of the synthesizer. [28] It is now possible to achieve frequency resolution that is a fractional portion of the phase detector frequency. This is accomplished by adding internal circuitry that enables the value of $N$ to change dynamically during the locked state. If the value of the divider is "switched" between $N$ and $N+1$ in the correct proportion, an average division ratio can be realized that is $N$ plus some arbitrary fraction, $K / F$. This allows the phase detectors to run at a frequency that is higher than the synthesizer channel spacing.

$$
f_{\text {out }}=\left(I N T+\frac{K}{F}\right) \times f_{\text {ref }}
$$

where, $F=$ the fractional modulus of the circuit, $K=$ the fractional channel of operation.

Figure 3.7 (b) shows the diagram of the fractional-N PLL frequency synthesizer. Comparison with 3.7 (a), it can be seen that fractional-N PLL allows larger reference frequency values, which results in a smaller multiplier term $N$. Since the PLL's phase noise is multiplied by the value of $N$ chosen and frac-N PLLs require smaller values for $N$, the phase noise increase due to multiplying the reference frequency by $N$ will decrease by $20 \log _{10}(N) \mathrm{dB}$. Another advantage is the smaller step-size or higher resolution. A frac-N allows step sizes on the order of tens of Hertz, while an integer-N may result in tens of kilohertz. The frac-N also will lock faster when compared to a similar integer-N solution. This is because the lower value of $\mathrm{N}$ allows a wider loop filter bandwith, which in turn allows a faster lock time. And the biggest disadvantages of a frac-N PLL are the fractional and integer boundary spurs it generates, its increased complexity from a usage standpoint. 


\subsubsection{Phase Noise Analysis}

Phase noise of PLL is a random process. Although we can use simulation software such as ADIsimPLL for analysis, we need to begin with the linearized PLL model, which is shown in figure 3.8 [29] :

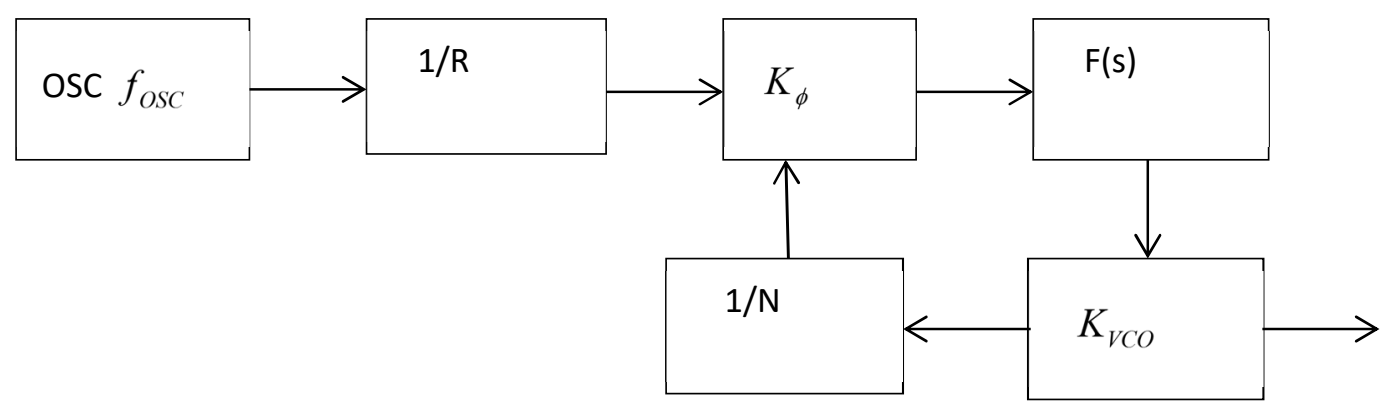

Figure 3.8 Linearized PLL model

Where $\mathrm{F}(\mathrm{s})$ is transfer function of loop filter, $K_{\phi}$ is phase detector sensitivity, $K_{V C O}$ is VCO sensitivity. From the PLL phase noise model, forward passage gain and reverse gain can be expressed as: $G(s)=\frac{K_{\phi} K_{V C O} F(s)}{s}$ and $H=\frac{1}{N}$.

We take the reference crystal oscillator as an example to derive the transfer function of each noise source: assume $\theta_{o s c}(s)$ is phase noise generated by reference crystal oscillator and ignore the input of other noise sources:

$$
\left(\frac{\theta_{\text {osc }}(s)}{R}-\frac{\theta_{\text {out }}(s)}{N}\right) K_{\phi} F(s) \frac{K_{V C O}}{s}=\theta_{\text {out }}(s)
$$

According to the equations of forward gain and reverse gain, the transfer function of reference crystal oscillator can be written as:

$$
T(s)=\frac{\theta_{\text {out }}(s)}{\theta_{\text {osc }}(s)}=\frac{1}{R} \frac{G(s)}{1+G(s) H}
$$

Table 3.1 Transfer function of noise components in PLL

\begin{tabular}{ll}
\hline Noise sources & Transfer function \\
\hline Crystal Oscillator & $\frac{1}{R} \frac{G(s)}{1+G(s) H}$ \\
R Divider & $\frac{G(s)}{1+G(s) H}$ \\
\hline
\end{tabular}




\begin{tabular}{ll}
\hline N Divider & $\frac{G(s)}{1+G(s) H}$ \\
Phase Detector & $\frac{1}{K_{\phi}} \frac{G(s)}{1+G(s) H}$ \\
VCO & $\frac{G(s)}{1+G(s) H}$ \\
\hline
\end{tabular}

From the table 3.1 shown above, we can notice that transfer functions of phase detector, R divider, $\mathrm{N}$ divider and crystal oscillator contain a same factor: $\frac{G(s)}{1+G(s) H}$. So these noise sources are collectively called in-band phase noise. To analyze this problem, phase margin $\phi$ are defined in equation (3.11), it determines stability as in other feedback loops:

$$
180^{\circ}-\angle G\left(j \omega_{o}\right) H=\phi
$$

Using these definitions and equations about forward passage gain and reverse gain, we can get, $\omega_{o}$ is the loop bandwidth:

$$
\frac{G(s)}{1+G(s)} \approx\left\{\begin{array}{cc}
N & \text { when } \omega<<\omega_{o} \\
G(s) & \text { whe } \mathrm{n} \omega>>\omega_{o}
\end{array}\right.
$$

Otherwise, the transfer function of VCO is $\frac{G(s)}{1+G(s) H(s)}$, and it can be expressed approximately as:

$$
\frac{G(s)}{1+G(s)} \approx \begin{cases}\frac{N}{G(s)} & \text { wh en } \omega<<\omega_{o} \\ 1 & \text { when } \omega>\omega_{o}\end{cases}
$$

Thus, as it shown in Figure 3.9, the in-band noise of PLL $\left(\omega<<\omega_{o}\right)$ mainly depends on crystal oscillator, phase detector and divider, and the out-band noise $\left(\omega>>\omega_{o}\right)$ depends on VCO. [30] 


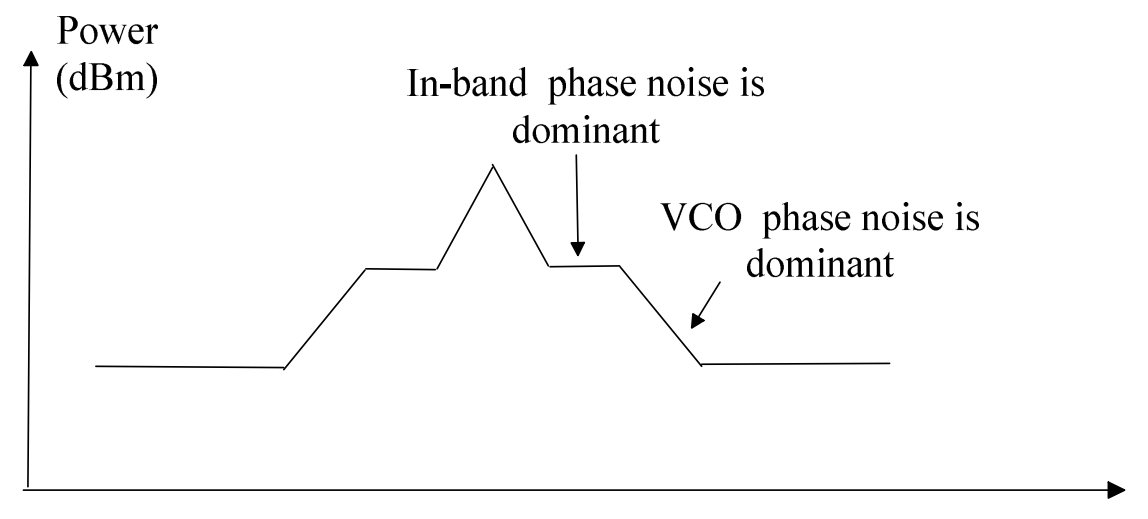

Figure 3.9 Phase noise spectrum of PLL

Besides, the frequency divider in the feedback path may have a significant contribution to the total phase noise of the PLL depending on its implementation and other properties of the loop. One of the cardinal principles is that multiplication by $\mathrm{N}$ causes loss of $20 \log _{10}(N)$ in phase noise performance; division improves by the same number. If a $100 \mathrm{kHz}$ crystal signal is multiplied by PLL (or any other way) to 1000 $\mathrm{MHz}$, the multiplication ratio is 10,000 and the corruption in phase noise will be $80 \mathrm{~dB}$. When divided down by a digital divider, the divider improves jitter by $\mathrm{N}$ times or $20 \log _{10}(N)$ in noise power.

In this thesis, we choose ADF4158 PLL chip, it is fractional-N PLL frequency synthesizer, of which RF bandwidth is up to $6.1 \mathrm{GHz}$, and it contains a 25-bit fixed modulus allowing subhertz resolution at $2.4 \mathrm{GHz}$. Figure 3.10 shows the triangle frequency modulation measured by real time analyzer DSO804A. From figure 3.10, we can find that ADF4158 PLL generates a continuous wave from 2.4 to $2.5 \mathrm{GHz}$ with a period of $1.06 \mathrm{~ms}$. 


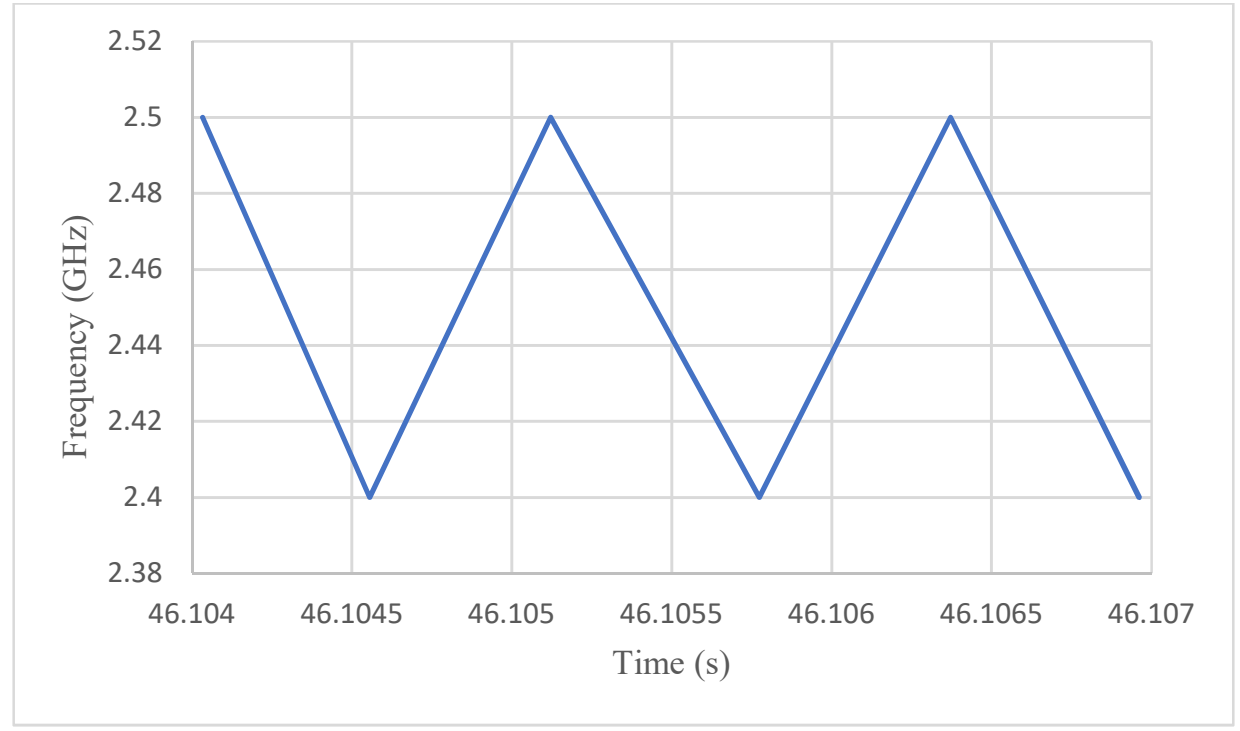

Figure 3.10 ADF4158 time domain measurement

Figure 3.11 show the output signal power spectra when $\mathrm{N}=192$ and $\mathrm{N}=1920$ : the loop bandwidth is $100 \mathrm{kHz}$ when $\mathrm{N}=192$ and loop bandwidth decreases to $20 \mathrm{kHz}$ when $\mathrm{N}$ increases to 1920 . We can find that the signal power spectrum with smaller $\mathrm{N}$ has wider loop bandwidth and better in band phase noise:

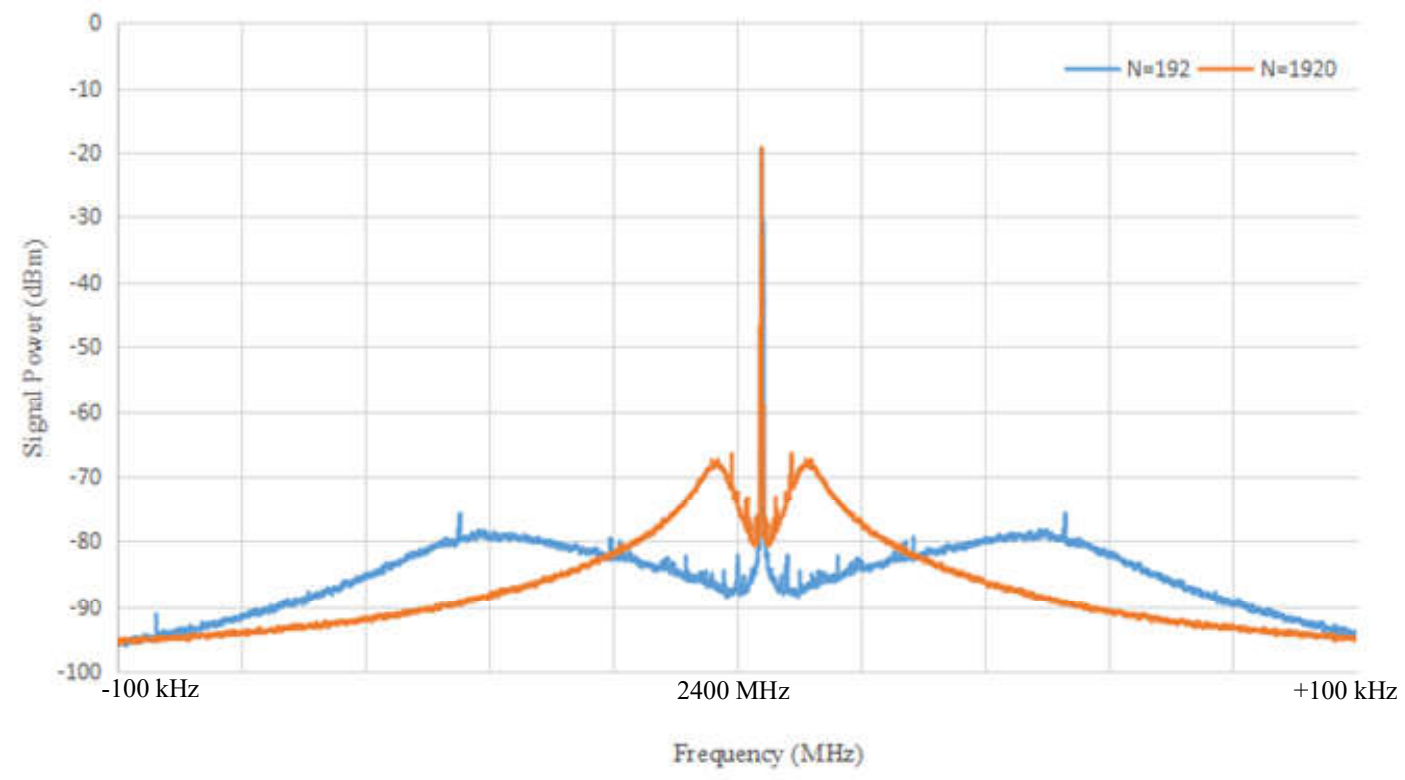

Figure 3.11 PLL power spectrum with different N

\subsection{DDS-based PLL Synthesizer}

Direct digital frequency synthesizer technology and phase-locked loop frequency 
synthesis technology have their own advantages and disadvantages. DDS has the advantage of quick switching time and low phase noise; the disadvantage is the low frequency, and the spectrum quality is poor. PLL is characterized by high output frequency, good quality spectrum; the drawback is the low frequency switching speed. The design requirements can be achieved by combining these two techniques considering bandwidth, frequency accuracy, switching time, phase noise, and spurs.

In this thesis, we use ADF 4158 evaluation board as the PLL frequency synthesizer and use AD9833 DDS board as reference frequency to replace the TCXO chip on PLL board. TCXO is a temperature compensated crystal oscillator. Compensation components have been added to enhance the temperature stability of the basic oscillator. Compared to TCXO, a major advantage of a DDS system is that its output frequency and phase can be precisely and rapidly manipulated under digital processor control. so that we can choose a high PFD frequency with good power spectrum for PLL.

Figure 3.15 shows the phase noise and different loop bandwidth with different $\mathrm{N}$ of ADF4158 PLL chip, the formula for the single sideband (SSB) phase noise in $\mathrm{dBc} / \mathrm{Hz}$ can be written as: $L(f m)=P_{S S B}(d B m)-10 \log (R B W)-P($ Carrier $)(d B m)$.

We can find that with the increase of $\mathrm{N}$, loop bandwidth decreases. As discussed in section 3.2.2 and 3.2.3, wider loop bandwidth means smaller in-band phase noise. Thus, for better phase noise, we set $f_{D D S}=f_{\text {ref }}=12.5 \mathrm{MHz}$ and $R=1$ in R counter and enable the reference doubler, thus the PFD frequency is $25 \mathrm{MHz}$. To generate frequency range from $2400 \mathrm{MHz}$ to $2500 \mathrm{MHz}$, the frequency synthesizer begins with:

$$
\left(96+\frac{0}{2^{25}}\right) \times 25 \mathrm{MHz}=2400 \mathrm{MHz}
$$

and ends with:

$$
\left(100+\frac{0}{2^{25}}\right) \times 25 \mathrm{MHz}=2500 \mathrm{MHz}
$$

As discussed in section 3.2, $\mathrm{N}$ causes increase of $20 \log _{10}(N)$ in phase noise performance. When $\mathrm{N}=96$, phase noise will increase about $40 \mathrm{~dB}$. Figure 3.14 shows in-band phase noise comparison between DDS and DDS-based PLL, we can find that the in-band phase noise of PLL is about $40 \mathrm{~dB}$ larger than phase noise of DDS. 


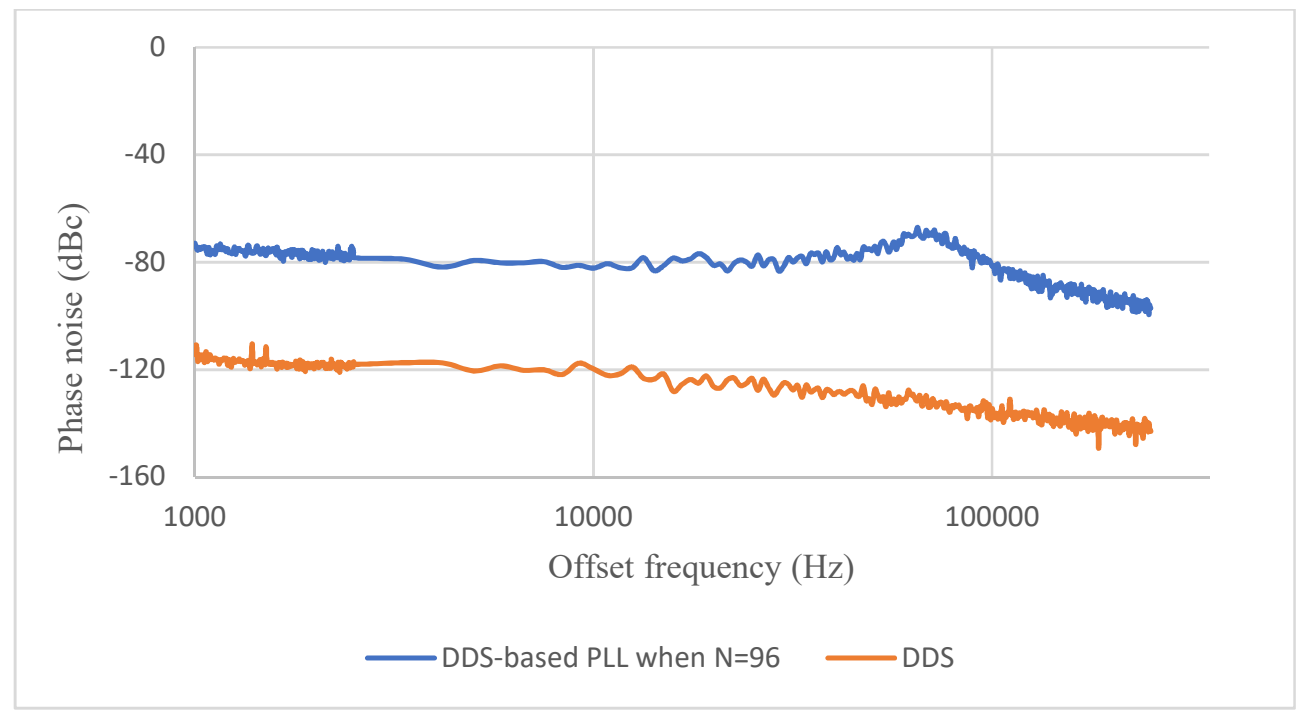

Figure 3.14 Phase noise comparison between DDS and DDS-based PLL when $\mathrm{N}=96$

$\mathrm{N}$ changes from 96 to 100 , and the minimum resolution is $f_{P F D} / 2^{25}=0.3725 \mathrm{~Hz}$. At this time, loop bandwidth is about $136 \mathrm{kHz}$ and phase noise is $-85 \mathrm{dBc} / \mathrm{Hz} @ 1 \mathrm{kHz}$.

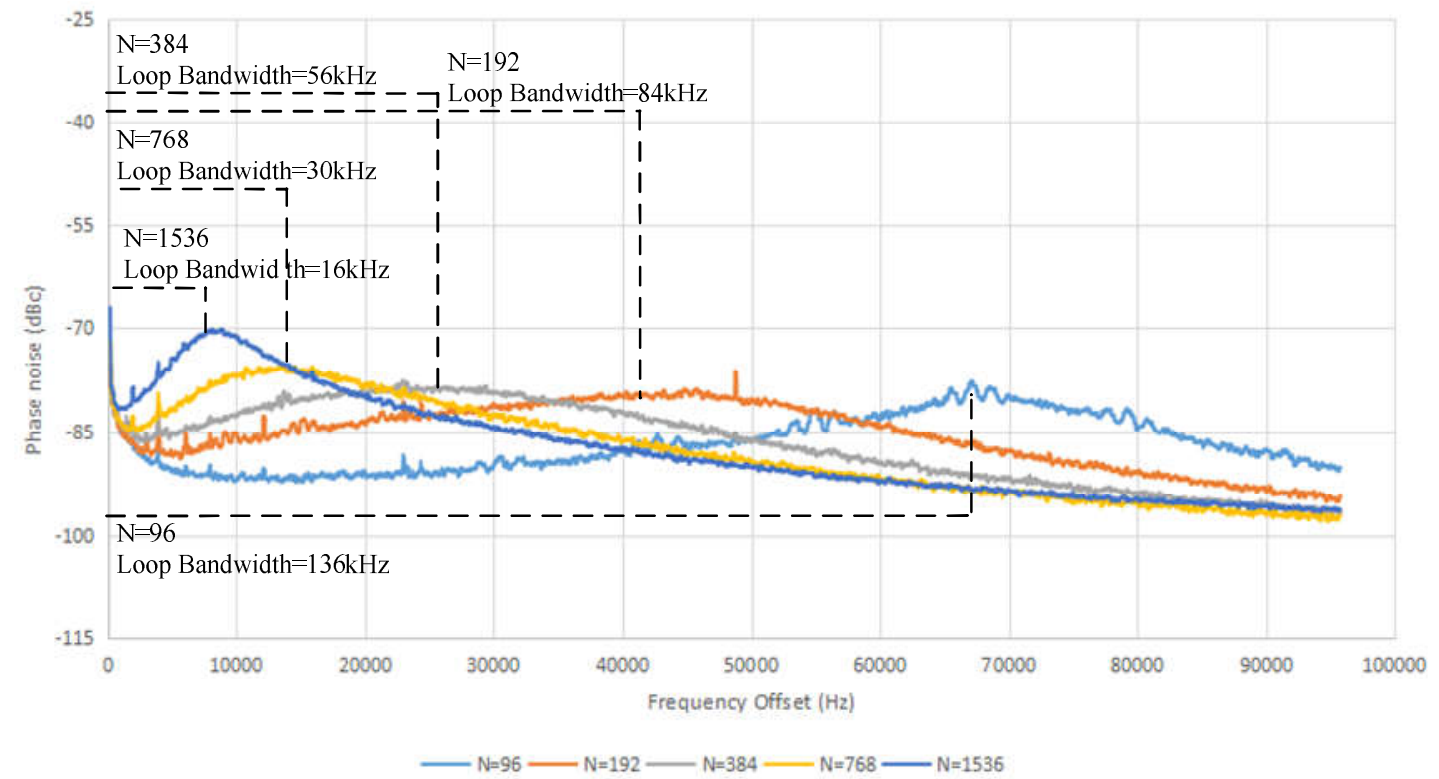

Figure 3.15 Phase noise measurement (measured by power spectrum analyzer Agilent E4440A, RBW=100 Hz, VBW=10 Hz)

As discussed in chapter 2, the IF bandwidth is $40 \mathrm{kHz}$, and PLL phase noise is about $-95 \sim-85 \mathrm{dBc} / \mathrm{Hz}$. And according to measurement in chapter 4, IF signal power is $-40 \mathrm{dBm}$ when object is $0.45 \mathrm{~m}$ far from the radar and IF power is about $-95 \mathrm{dBm}$ when $\mathrm{R}=10 \mathrm{~m}$. Thus, such phase noise is much smaller than IF power when object is 
close. And phase noise sources in a primary radar (phase noise from RF and LO signals) are correlated and as a result, the phase noise will partially cancels out due to range correlation effects as shown in [24]. So in chapter 4, the thesis will focus on how receiver system noise and gain influence the performance of the radar transceiver.

\subsection{Summary}

The comparative discussion consists of PLL, DDS and DDS- based PLL. We discuss the working principle of DDS and PLL, and a DDS-driven PLL frequency synthesizer architecture is given in this chapter. We also talk about some main parameters for frequency synthesizer, particularly phase noise and spurs. And do the measurement about phase noise of PLL board and DDS-based PLL board. The measurement proves that the design of DDS + PLL frequency synthesizer adopts DDS driving PLL generate RF signals and the generated frequency is stable, reliable. The signal amplitude and phase noise can be up to the requirements of FMCW radar transceiver. Next the DDS+PLL frequency synthesizer is put into use. 


\section{Chapter 4}

\section{FMCW Radar System Design and Test}

In this thesis, we use a homodyne receiver with one output signal. After being amplified by a power amplifier, the transmitting signal is received by the homodyne receiver: incoming RF signal is down-converted to baseband (zero frequency) in one step by mixing with an oscillator output of the same frequency. The resulting baseband signal is then filtered with a low-pass filter to select the desired channel. To improve the input and output matching of LNA, we add two isolators in the receiver. This is illustrated in the block diagram in figure 4.1.

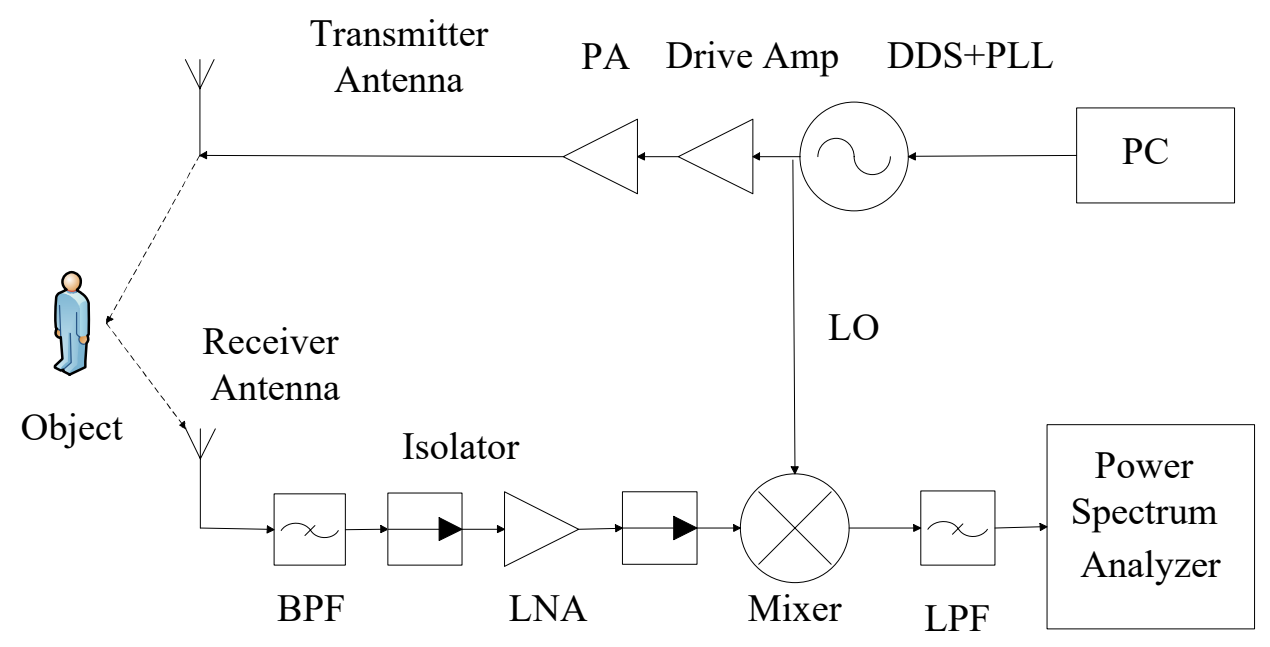

Figure 4.1 Simplified block diagram of radar transceiver

This thesis calculates distance information by IF signals from power spectrum analyzer instead of FFT. And this radar still shows the good performance to position and track objects in short range. 


\subsection{Radar System Design}

\subsubsection{LNA design and test}

In this thesis, we describe how to design a microwave LNA at $2.4 \mathrm{GHz}$ starting from a BJT transistor chip to the actual board layout. The transistor used is the Freescale Semiconductor Transistor, part BFU760F, which is a NPN wideband silicon germanium $\mathrm{RF}$ transistor. This transistor is characterized as follows at the quiescent operating point with targeted frequency of $2.4 \mathrm{GHz}$. The load line and S matrix are determined through simulations. This resulted in unilateral operation of the transistor which implies a simpler matching network because there is limited internal transistor feedback. Source and load matching networks are designed at $50 \mathrm{Ohms}$ with the corresponding $\mathrm{S}$ matrix. Stability was simulated and proven to be stable for the specified biasing range of the part. In the measurement shown in figure 4.2, the gain of our amplifier circuit is $16.4 \mathrm{~dB}$ at $2.45 \mathrm{GHz}$, which is close to the simulation. Noise figure is measured by spectrum analyzer and a noise source NC3101. At $2.5 \mathrm{GHz}, \mathrm{NF}$ is $2.3 \mathrm{~dB}$.

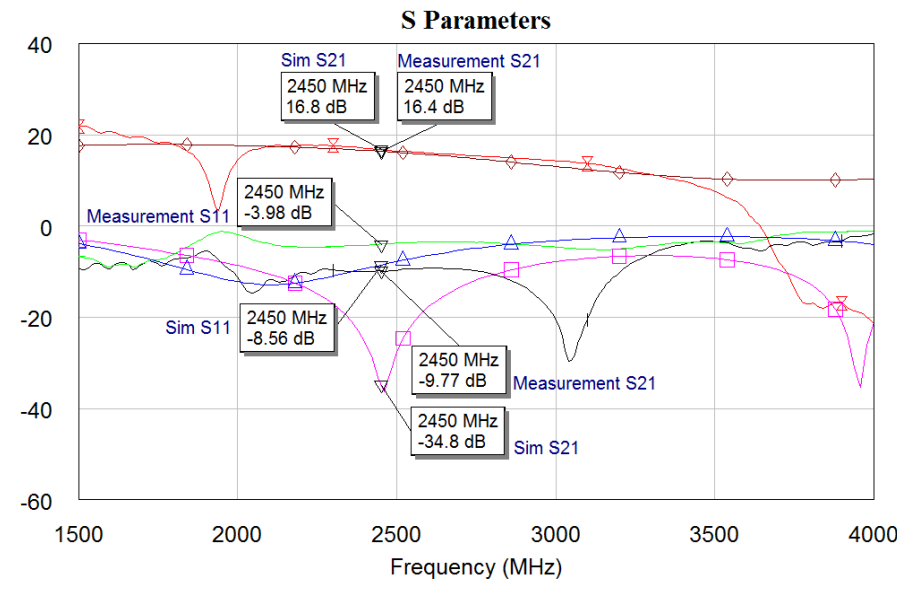

(a) S parameters of LNA

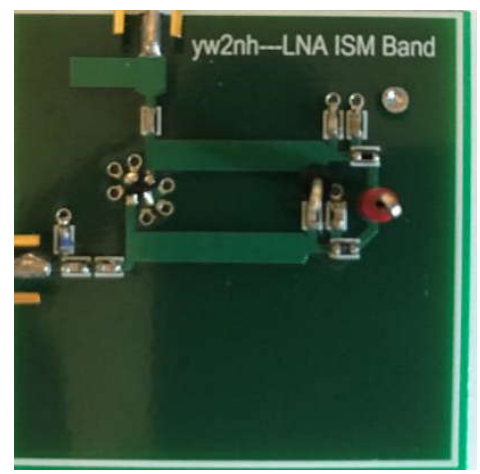

(b) Picture of LNA

Figure 4.2 Measurement of LNA 


\subsubsection{Filter design and test}

The low pass filter only allows low frequency signals from $0 \mathrm{~Hz}$ to its cut-off frequency to pass while blocking those any higher. In this thesis, we design a maximally flat low-pass filter with a cutoff frequency of $40 \mathrm{kHz}$, impedance of $50 \mathrm{Ohm}$, and at least $10 \mathrm{~dB}$ insertion loss at $50 \mathrm{kHz}$ and $40 \mathrm{~dB}$ insertion loss at $100 \mathrm{kHz}$.

The high pass filter only allows high frequency signals from its cut-off frequency, while blocking those any lower. And the band pass filter allows signals falling within a certain frequency band setup between two points to pass through while blocking both the lower and higher frequencies either side of this frequency band, [25]. The parallel coupled transmission lines are used to construct band-pass filter, whose the passband is 2.4 GHz to $2.5 \mathrm{GHz}$. The results of two filters are shown in figure 4.3.

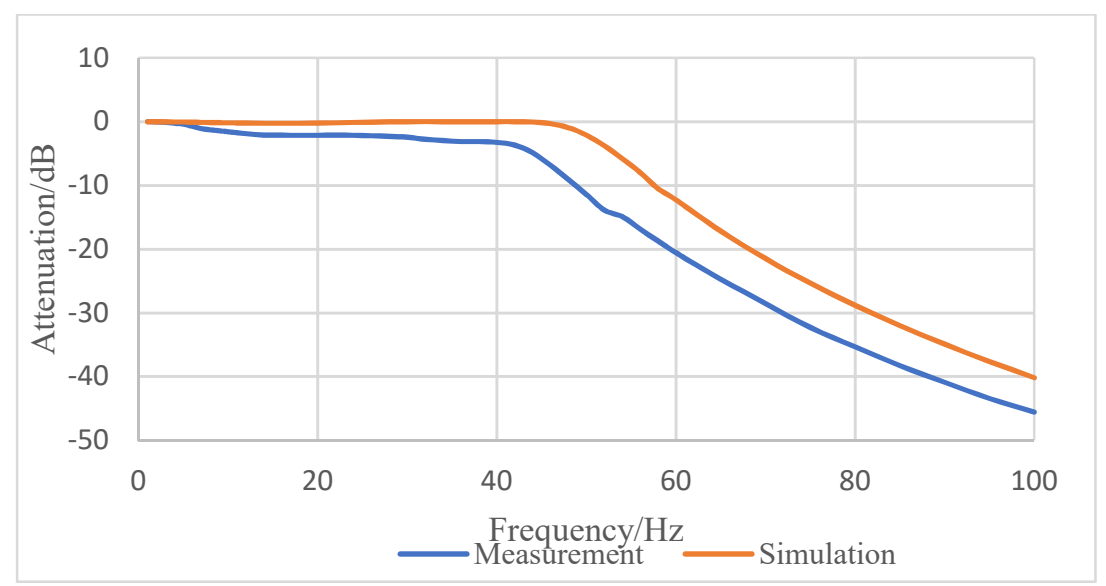

(a) Attenuation of LPF

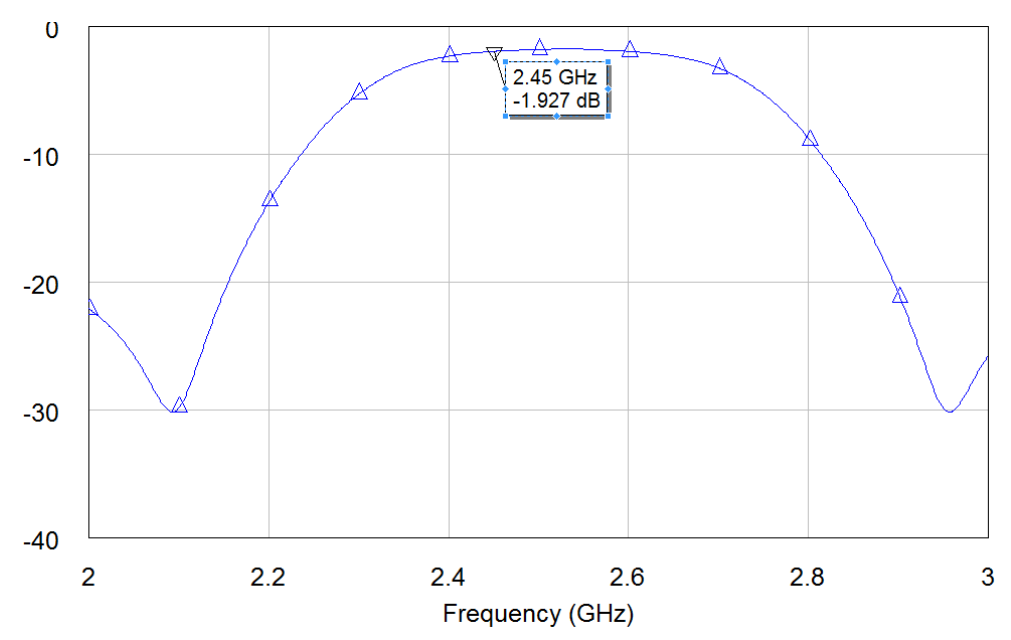

(b) Attenuation of BPF 


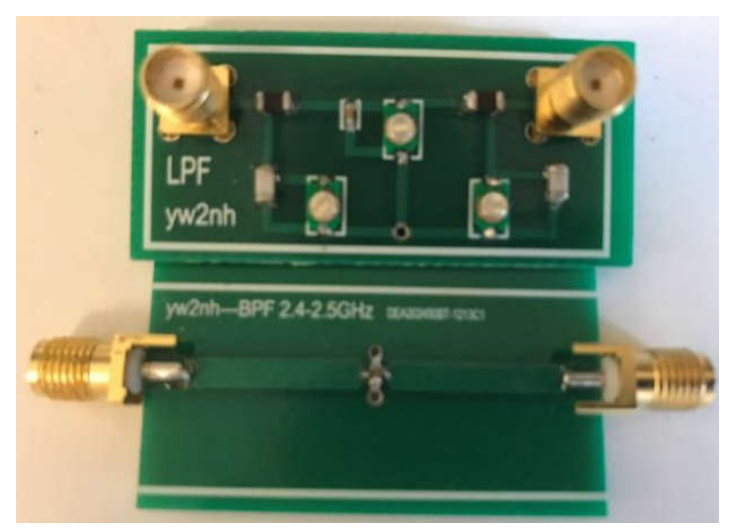

(c) Picture of LPF and BPF

Figure 4.3 Filter attenuation

\subsubsection{Mixer Test}

A mixer is a three-port device that uses a nonlinear or time-varying element to achieve frequency conversion. An ideal mixer produces an output consisting of the sum and difference frequencies of its two input signals. Operation of practical RF and microwave mixers is usually based on the nonlinearity provided by either a diode or a transistor. In this thesis, we need a frequency down-conversion mixer in the radar receiver, the desired IF output in a receiver is the difference frequency, which can be easily selected by low-pass filtering: $f_{I F}=f_{R F}-f_{L O}$.

This work adopts a double-balanced mixer, which uses two hybrid junctions or transformers, and provides good isolation between all three ports, as well as rejection of all even harmonics of the RF and LO signals. [30] This leads to very good conversion loss, but less than ideal input matching at the RF port. The double-balanced mixer also provides a higher third-order intercept point than either a single-ended mixer or a balanced mixer.

An important figure of merit for a mixer is therefore the conversion loss, which is defined as the ratio of available RF input power to the available IF output power, expressed in $\mathrm{dB}$ :

$$
L_{c}(d B)=P_{R F}-P_{I F}
$$

In this thesis, we use LTC5548 microwave double balanced passive mixer, its main parameters are shown in table 4.1: 


\begin{tabular}{ll}
\hline Parameters & Values \\
\hline Conversion Loss & $6.2 \mathrm{~dB} @$ RF input $=2.4 \mathrm{GHz}$ \\
Noise Figure & $7 \mathrm{~dB} @ \mathrm{RF}$ input $=2.4 \mathrm{GHz}$ \\
Input P1dB Compression & $16 \mathrm{dBm}$ \\
LO to RF Leakage & $<-25 \mathrm{dBm}$ \\
LO to IF Leakage & $<-25 \mathrm{dBm}$ \\
\hline
\end{tabular}

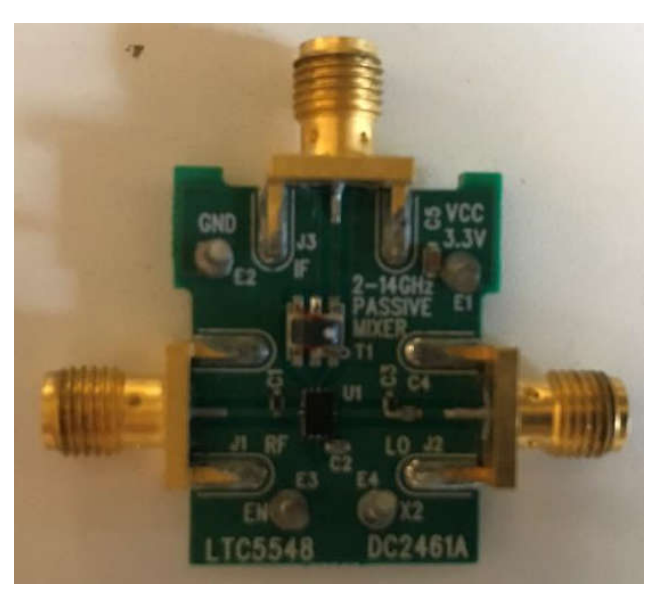

Figure 4.4 Picture of mixer

\subsubsection{Power Amplifier Test}

RF power amplifiers are used to increase the output power in a wide variety of applications including wireless communication, TV transmissions, radar, and RF heating. The thesis adopts the MMG3H21NT1 broadband high linearity amplifier chip and TQP9111 power amplifier chip. MMG3H21NT1 is a general purpose amplifier, which has $12.6 \mathrm{~dB}$ small-signal gain, $5.4 \mathrm{~dB}$ input return loss and $10.4 \mathrm{~dB}$ output return loss. TQP9111 is a high efficiency two-stage driver amplifier in a low-cost surfacemount package. This linear amplifier integrates two high performance amplifier stages onto a module to allow for a compact system design. The integrated inter-stage match minimizes performance variation that would otherwise be attributed to external matching component value and placement tolerances. At $2.4 \mathrm{GHz}$, this PA has $13 \mathrm{~dB}$ input return loss, $20 \mathrm{~dB}$ output return loss, $29.8 \mathrm{~dB}$ gain and $32.5 \mathrm{dBm} 1 \mathrm{~dB}$ compression point. 


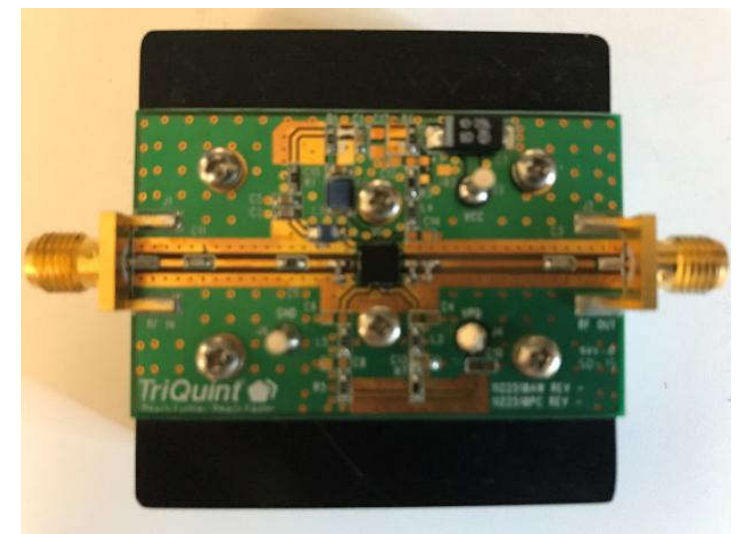

(a) Power amplifier

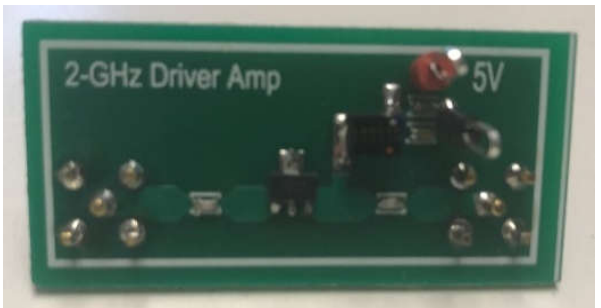

(b) Drive amplifier

Figure 4.5 Picture of amplifiers

\subsubsection{Antenna Test}

In this radar system, we adopt two monopole antennas as transmitting and receiving antennas. Main parameters of the antennas are shown in table 4.2. Return loss is measured by VNA. Antenna gain is cited in the datasheet of monopole antenna GW.05.0153.66666

Table 4.2 Main Parameters of antenna

\begin{tabular}{llll}
\hline & $2400 \mathrm{MHz}$ & $2450 \mathrm{MHz}$ & $2500 \mathrm{MHz}$ \\
\hline Average gain (dBi) & -2.62 & -2.61 & -1.74 \\
Efficiency (\%) & 54.71 & 54.78 & 67.05 \\
Peak gain (dBi) & 1.04 & 1.25 & 0.82 \\
Return loss $(\mathrm{dB})$ & $<-6$ & $<-6$ & $<-6$ \\
\hline
\end{tabular}

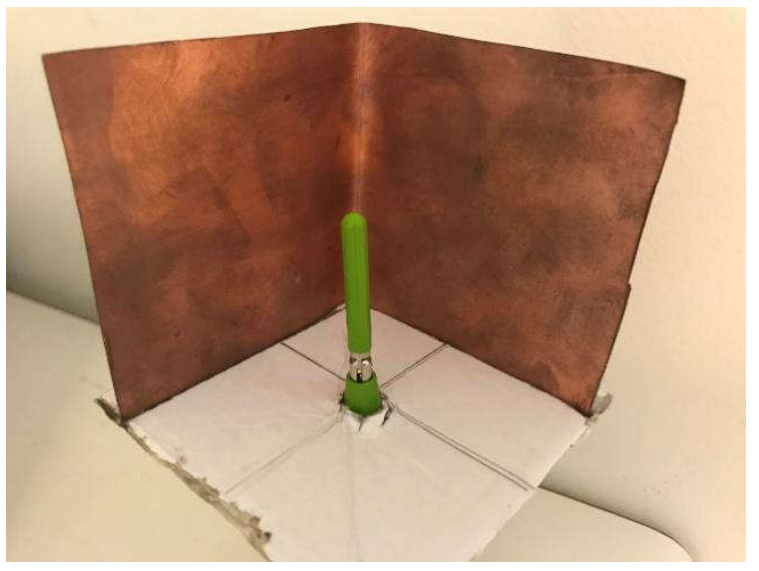

Figure 4.6 Picture of antenna 


\subsubsection{Radar Components Summary}

In this chapter, we designed each component of the homodyne radar receiver, including low-noise amplifier, band-pass filter, low-pass filter, mixer and power amplifier, the summary is shown in table 4.3. In this system, total noise and gain is shown below:

$$
N F_{\text {total }}=10 \log _{10}\left(n_{1}+\sum_{i=2}^{3} \frac{n_{i}-1}{\prod_{j=1}^{i-1} g_{j}}\right)=3.529 d B \quad G_{\text {total }}=\sum G_{i}=9.2 d B
$$

Table 4.3 Summary of Radar Components

\begin{tabular}{ll}
\hline Component Name & Main Parameters \\
\hline Low-noise amplifier & $\mathrm{S} 11=-3.98 \mathrm{~dB} \quad \mathrm{~S} 22=-9.77 \mathrm{~dB}$ \\
& $\mathrm{~S} 21=16.4 \mathrm{~dB}$ \\
& $\mathrm{NF}=2.3 \mathrm{~dB} @ 2.45 \mathrm{GHz}$ \\
& Passband=2.4-2.5 GHz Loss=1.93 dB \\
Band-pass filter & Passband=0-40 kHz \\
Low-pass filter & Down Converter \\
Mixer & $\mathrm{NF}=7 \mathrm{~dB}$ \\
& Conversion Loss $=6.2 \mathrm{~dB} @ 2.45 \mathrm{GHz}$ \\
& $\mathrm{S} 11=-5.4 \mathrm{~dB} \quad \mathrm{~S} 22=-10.4 \mathrm{~dB}$ \\
Drive Amplifier & $\mathrm{S} 21=12.6 \mathrm{~dB} @ 2.45 \mathrm{GHz}$ \\
& Power gain=29.8 dB \\
Power Amplifier & $\mathrm{S} 11=-13 \mathrm{~dB}, \mathrm{~S} 22=-20 \mathrm{~dB} @ 2.45 \mathrm{GHz}$ \\
& Loss=0.18 dB \\
Isolator & Average gain=-2.62 dBi @2.4 GHz \\
Antenna & Return loss $<-6 \mathrm{~dB}$ \\
\hline
\end{tabular}




\subsection{Human indoor positioning and tracking}

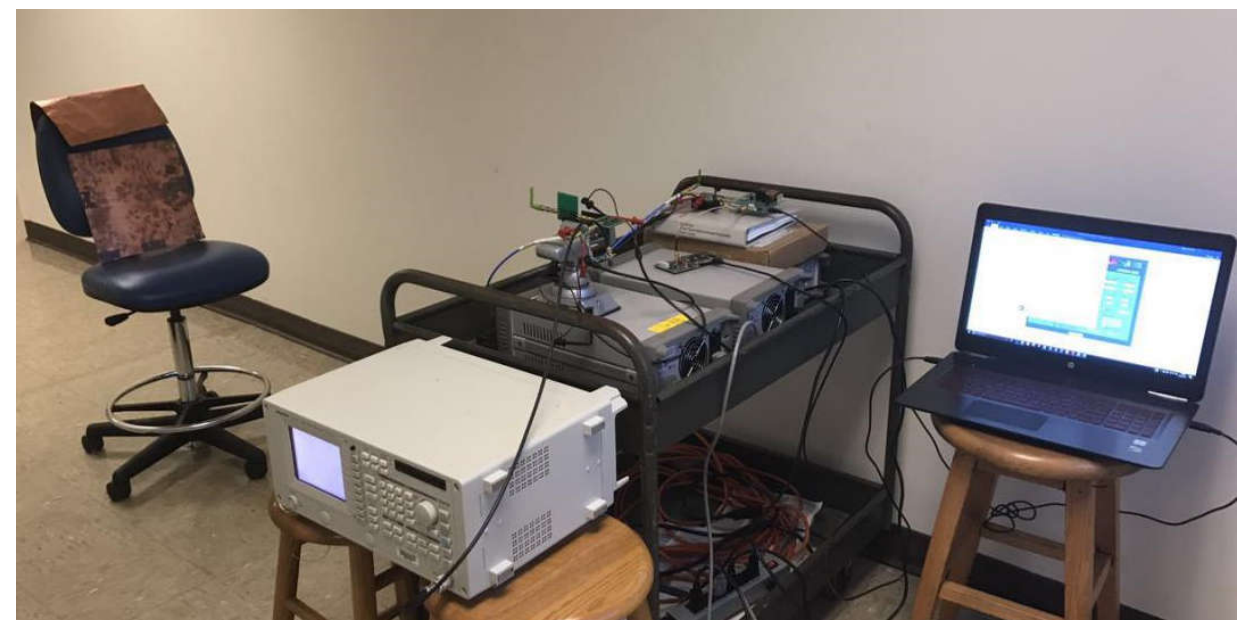

Figure 4.7 Radar test in the aisle in Thornton Hal

The radar receiver is tested in the aisle in Thornton Hall, and the attenuation and reflection of wall and electrical devices will affect the results, particularly when the transmitting signal is weak.

In this test, we use a power spectrum analyzer Agilent E4440A to measurement. The measurement range is from $-5-40 \mathrm{kHz}$ and there are 600 samples in this range. Figure 4.8 with blue line shows the IF power spectrum when there is no objective in the front of radar system. It can be seen that there are spurs in the spectrum resulting from reasons shown below:

(1) Receiver mismatch: The input and output return loss of LNA is not very good, which means that signal may bounce between LNA and BPF. Such oscillation means there will be more than one RF frequency for the mixer RF input, and finally results in inter-modulation of the mixer. Besides, the receiver may suffer even-order distortion. When there are two strong frequencies $\omega_{1}$ and $\omega_{2}$ at the input of LNA, it will generate a low-frequency beat $\omega_{1}-\omega_{2}$. Since the isolation is finite from the RF input to the IF output, such low-frequency beat will appear in the IF port.

When the LNA is removed, we can find that spurs still exist but are decreased significantly. Besides, when we use lossless cables to replace antennas and objects, the power of spurs increases. If we add an attenuator at the input of BPF, the spurs power decreases. Thus, it can be proved that the radar receiver generates spurs. To solve this problem, we add two isolators at the input and output of LNA. The comparison between IF frequency without isolators and IF frequency with isolators is shown in figure 4.8. 
The red curve is power spectrum after adding isolators, we can find that the quality of spectrum improves because of isolators.

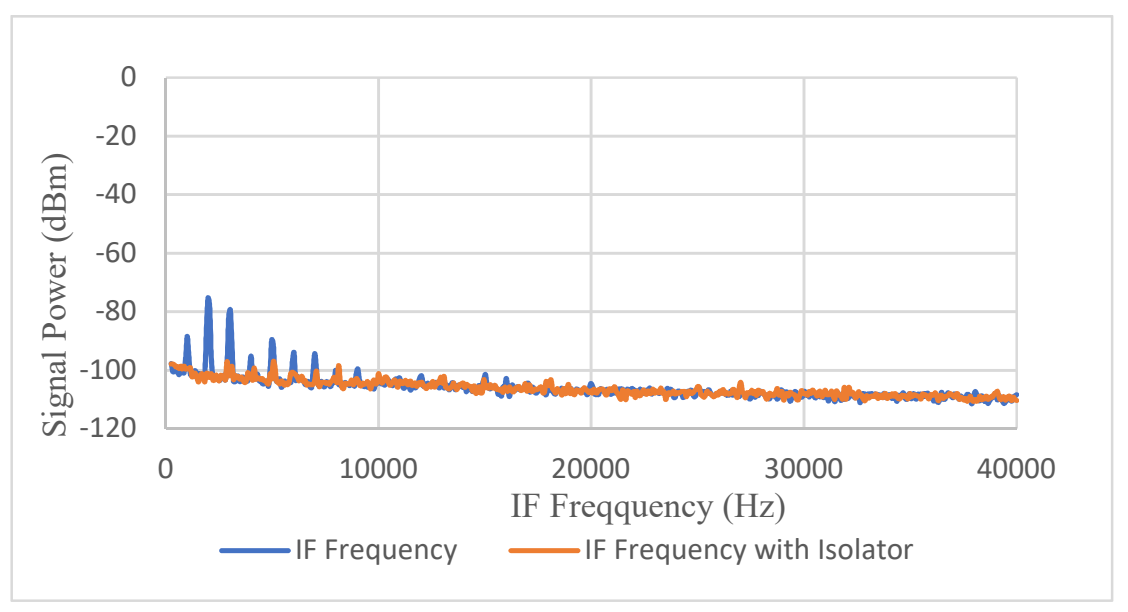

Figure 4.8 Spectrum comparison between IF with isolators and IF without isolators

(2) LO leakage and DC offset: When the isolation between the LO port and the inputs of the mixer is not infinite, LO signals can leak to mixer RF port and LNA input, finally leak to the receiving antenna. Such LO leakage is received by mixer RF port and mixed with LO. It is called DC offset because such frequency beat is zero. When the transmitting antenna is removed and the radar receiver works (no RF signal), spurs decreases but still exist, which is shown in figure 4.9. Thus, LO leakage can generate spurs, but it is not the main reason in this system.

Besides, we also need to consider LO phase noise, [37]. The noise source of LO and RF signals are correlated and as a result, the phase noise partially cancels out due to range correlation effects.

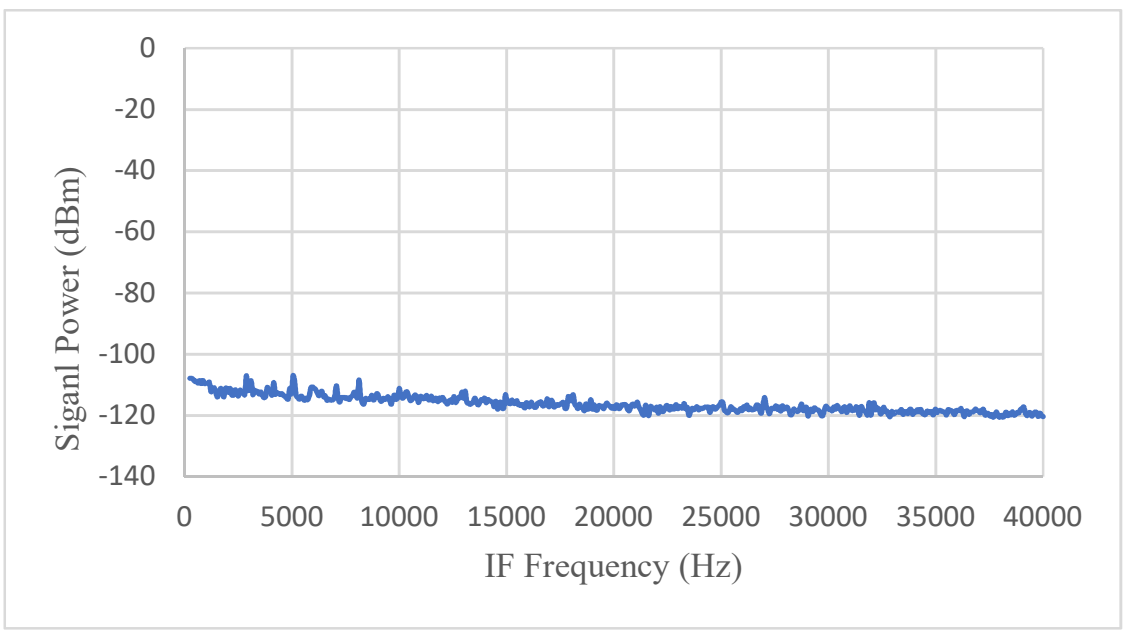

Figure 4.9 IF spectrum with LO leakage (antennas are replaced by $50 \Omega$ termination) 
(3) Reflection: the reflection from the environment can also generate spurs. The radar system is tested in an empty aisle for weak reflection. But multireflection still generates spurs, particularly when the object is close to radar system. Multireflection can generate more than one RF signal and result in mixer inter modulation: when more than one RF signals, $f_{1}$ and $f_{2}$, go into mixer RF port, spurs $\pm\left[\left(r f_{1}-s f_{2}\right)-f_{L O}\right]$ will be generated periodically.

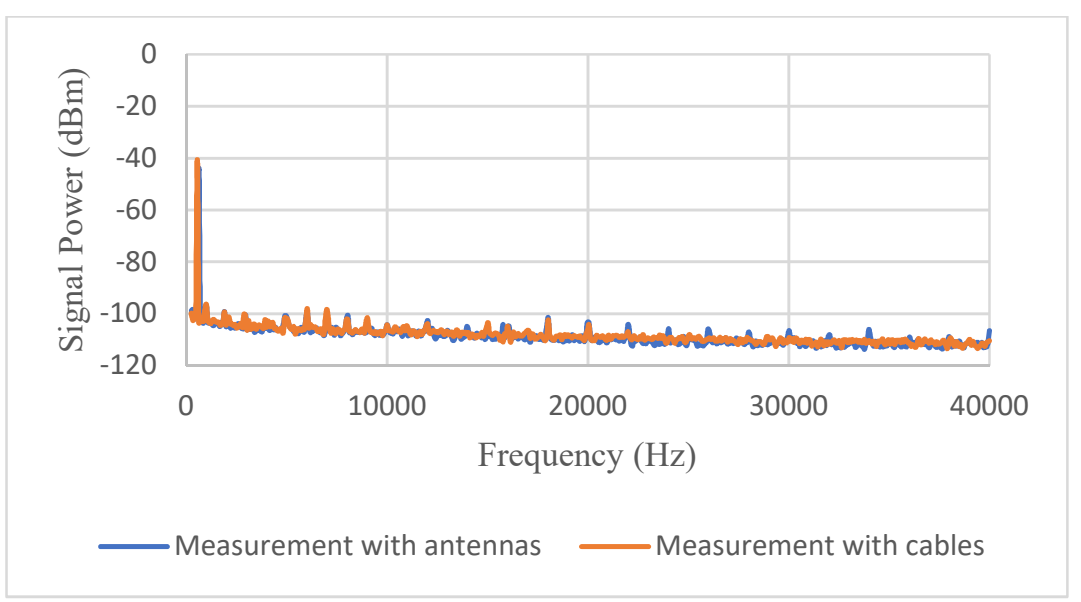

(a) IF signal when $R=0.45 \mathrm{~m}$ measured in the aisle $\left(I F=600 \mathrm{~Hz}, I F_{\text {Measurement }}=625 \mathrm{~Hz}\right)$

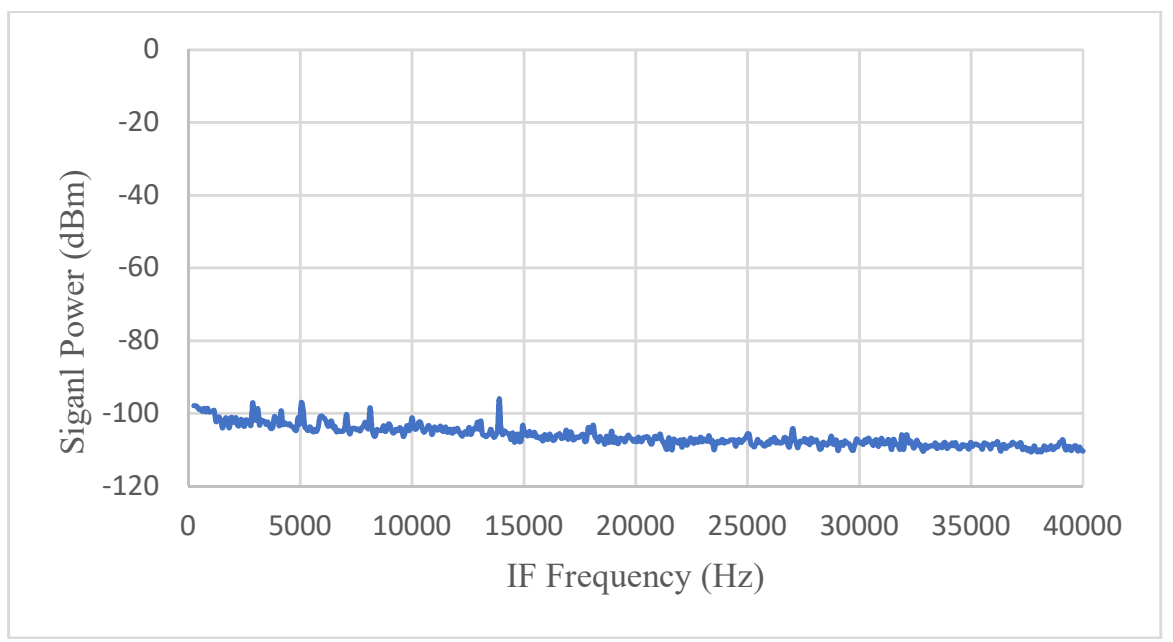

(b) IF signal when $R=10 \mathrm{~m}$ in the aisle

$$
\left(I F=13333 \mathrm{~Hz}, I F_{\text {Measurement }}=13600 \mathrm{~Hz}\right)
$$

Figure 4.10 IF signal spectrum

Figure 4.10 (a) and (b) shows this IF power difference when $R=0.45 \mathrm{~m}$ and $R=10$ m. The thesis makes a control experiment: a person stands in the front of radar transceiver and holds a copper plate to enhance reflection, the IF power spectrum is 
recorded after the result is stable. After that, we use cables with the same distance to measure it again, the IF spectrum in the "pure environment" is recorded again. The two data can be compared to check the accuracy of the radar. All data can be checked in appendix. After range is larger than $10 \mathrm{~m}$, the effective IF signal is inundated in spurs and noise. After calculation, we can find that there is difference between calculation and real range information. With the increase of range, such error increases and positioning accuracy decreases. Figure 4.11 plots the error distribution with 10 times measurement, as shown in the figure, the maximum error for all the tests is about 30 $\mathrm{cm}$, and maximum standard deviation is $7 \mathrm{~cm}$ (The y axis for distance is on the left and the $\mathrm{y}$ axis for standard deviation is on the left). This distance detection accuracy is sufficient for indoor localization purposes.

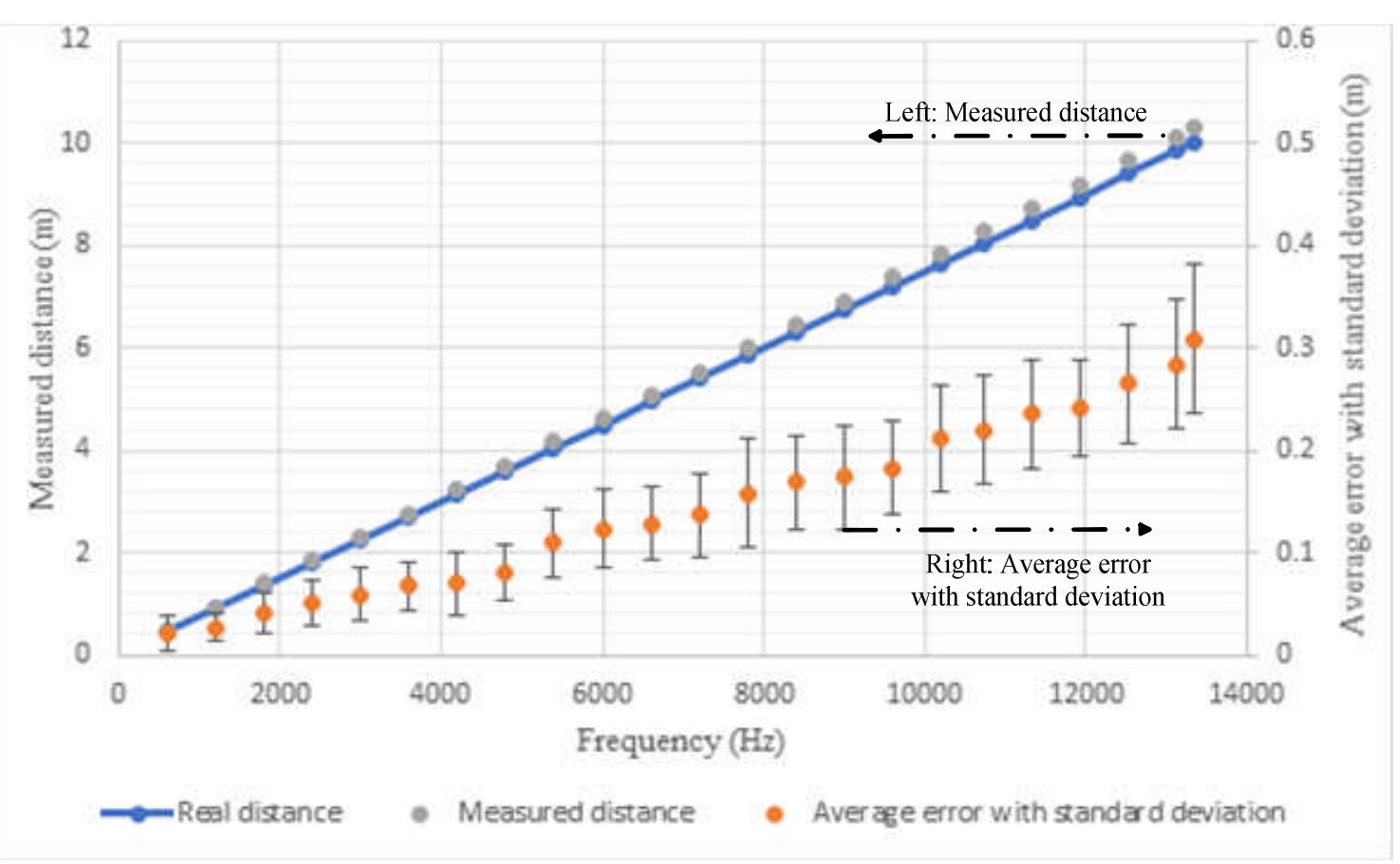

Figure 4.11 Range measurement

\subsection{Analysis and Future Work}

It's necessary for us to discuss about ideal maximum detection range: the radar range equation represents the physical dependences of the transmit power, that is the wave propagation up to the receiving of the echo-signals. 


$$
R_{\max }=\sqrt[4]{\frac{P_{t} G_{t} G_{r} \lambda^{2} \sigma}{(4 \pi)^{3} P_{\min }}}
$$

where $P_{t}$ is transmit power, $G_{t}$ and $G_{r}$ are transmitting and receiving antenna gain, $\lambda$ is transmit wavelength, $\sigma$ is target radar cross section, $P_{\min }$ is the minimum detectable signal.

The antenna gain is $-2 \mathrm{dBi}$, wavelength is $0.12 \mathrm{~m}$ and radar cross section is a $0.3 \times 0.3 \mathrm{~m}$ copper plate. $P_{\min }=k\left(T_{a}+T_{\text {receiver }}+T_{\text {analyzer }}\right) B \cdot(S N R)_{\min }$, where $T_{a}$ is antenna noise temperature, $T_{\text {receiver }}$ is receiver noise temperature and $T_{\text {analyzer }}$ is spectrum analyzer noise temperature.

Since it's hard to calculate antenna noise temperature, we design an experiment to measure it, which is shown in figure 4.10. First, we connect the noise source NC3011 to spectrum analyzer to get noise power $P_{o}$, and then turn on the noise source to get noise power $P_{H}$. We can get ENR of the noise source from data sheet and calculate noise temperature of noise source $T_{\text {noise }}$ :

$$
E N R=\frac{T_{\text {noise }}}{T_{o}}-1=15.29 \mathrm{~dB}=33.8 \rightarrow T_{\text {noise }}=34.8 T_{o}
$$

where $T_{o}$ is room temperature.

And we can get $\mathrm{Y}$ factor:

$$
Y=\frac{P_{H}}{P_{o}}=3.64
$$

Thus, noise temperature of spectrum analyzer is:

$$
\begin{gathered}
F_{\text {analyzer }}=\frac{E N R}{Y-1}=12.8=11.1 \mathrm{~dB} \\
T_{\text {analyzer }}=\left(F_{\text {analyzer }}-1\right) T_{o}=11.8 T_{o}
\end{gathered}
$$

Note that the analyzer shown in (4.6) is measured at $2.4 \mathrm{GHz}$ and cannot be used in radar equation (4.2) because the IF frequency is $0-40 \mathrm{kHz}$.

Then we use antenna to replace noise source and get noise power $P_{H}{ }^{\prime}$. Since we use the same resolution bandwidth and reference level, we can get: 


$$
\begin{aligned}
& \frac{P_{H}^{\prime}}{P_{H}}=\frac{k\left(T_{\text {antenna }}+T_{\text {analyzer }}\right) B}{k\left(T_{\text {noise }}+T_{\text {analyzer }}\right) B}=\frac{T_{\text {antenna }}+T_{\text {analyzer }}}{T_{\text {noise }}+T_{\text {analyzer }}} \\
& \Rightarrow T_{\text {antenna }}=\frac{P_{H}^{\prime}}{P_{H}}\left(T_{\text {noise }}+T_{\text {analyzer }}\right)-T_{\text {analyzer }}=9.17 T_{o}
\end{aligned}
$$

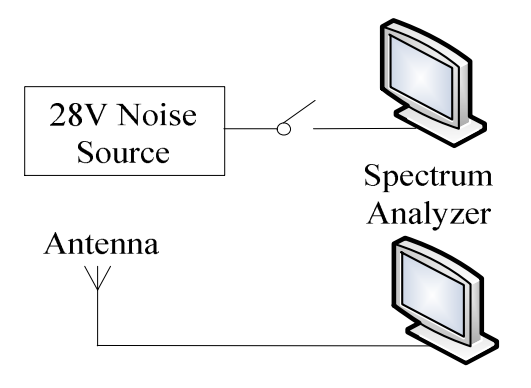

Figure 4.10 Antenna temperature measurement

Next, we need to discuss about parameters of spectrum analyzer since we use the spectrum analyzer instead of FFT algorithm: Resolution bandwidth also affects sensitivity. The ideal RBW has a flat passband and infinite attenuation outside that passband. But it must also have good time domain performance so that it behaves well when signals sweep through the passband. The spectrum analyzer in this thesis uses four-pole synchronously tuned filters for their RBW filters. The response of the filter to noise of flat power spectral density will be the same as the response of a rectangular filter with the same maximum gain and the same area under their curves. The width of such a rectangular filter is called the equivalent noise bandwidth of the RBW filter. The ratio of the equivalent noise bandwidth to the resolution bandwidth is 1.128.

The input attenuator also affects the location of a true input signal on the display. Signals present at the analyzer input remain stationary on the display as we change the input attenuator, while the displayed noise moves up and down. Thus, we get the best sensitivity by selecting minimum input attenuation.

The video filter can be used to reduce the amplitude fluctuations of noisy signals while at the same time having no effect on constant signals. It should be noted that the video filter does not affect the average noise level and so does not, by this definition, affect the sensitivity of an analyzer.

Then we assume $S N R_{\min }$ is $5 \mathrm{~dB}$, and $T_{\text {antenna }}=9.17 T_{o}, T_{\text {receiver }}=1.25 T_{o}$ calculated by noise figure of receiver. Since we don't have noise source at $40 \mathrm{kHz}$ and cannot know the noise temperature of spectrum analyzer at IF frequency, we use noise temperature at $2.4 \mathrm{GHz}$ to replace it. In this assumption, we can calculate the minimum 
power that the antenna can receive:

$$
\left(P_{\text {in }}\right)_{\min }=k\left(T_{a}+T_{\text {receiver }}+T_{\text {analyzer }}\right) \cdot B \cdot(S N R)_{\min }
$$

Thus, the minimum power that the spectrum analyzer can receive is:

$$
\left(P_{\text {out }}\right)_{\min }=\left(P_{\text {in }}\right)_{\min }(d B m)+G(d B)=-140 d B m
$$

Thus, according to radar equation (4.2), we can calculate the maximum detectable range. $R_{\max }$ is $20 \mathrm{~m}$ when $\left(P_{\text {out }}\right)_{\min }=-140 \mathrm{dBm}$.

In the measurement, the maximum range is $10 \mathrm{~m}$. The difference between calculation and measurement is resulted by assumed noise temperature and spectrum analyzer. Large RBW will increase the noise floor of the spectrum analyzer. Table 4.4 makes a summary of some problems and components which affect maximum detection range. Since we cannot get the precise value of ideal maximum detectable range, it is still necessary to analyze these parameters.

\begin{tabular}{|c|c|c|c|}
\hline & Components & Description & Solution \\
\hline \multirow[t]{4}{*}{$\begin{array}{l}\text { Spectrum } \\
\text { Analyzer }\end{array}$} & Attenuator & $\begin{array}{l}\text { Input signals remain stationary } \\
\text { and displayed noise moves up } \\
\text { with the increase of attenuation. }\end{array}$ & $\begin{array}{l}\text { Set minimum } \\
\text { attenuation } 0 \mathrm{~dB}\end{array}$ \\
\hline & $\begin{array}{l}\text { Resolution } \\
\text { Bandwidth } \\
\text { (RBW) }\end{array}$ & $\begin{array}{l}\text { The bandwidth of a flat band- } \\
\text { pass filter. Smaller RBW is, } \\
\text { higher frequency resolution is. }\end{array}$ & $\mathrm{RBW}=75 \mathrm{~Hz}$ \\
\hline & $\begin{array}{l}\text { Video } \\
\text { Bandwidth }\end{array}$ & $\begin{array}{l}\text { The video filter reduces the } \\
\text { amplitude fluctuations of noisy } \\
\text { signals but doesn't affect noise } \\
\text { level. }\end{array}$ & $\mathrm{VBW}=10 \mathrm{~Hz}$ \\
\hline & DC Offset & $\begin{array}{l}\text { DC Offset is an offseting of a } \\
\text { signal from zero. }\end{array}$ & $\begin{array}{l}\text { Blocking } \\
\text { Capacitor }\end{array}$ \\
\hline \multirow[t]{3}{*}{ Receiver } & Mismatch & $\begin{array}{l}\text { When input and output return } \\
\text { loss is not good, signal oscillates } \\
\text { between components. }\end{array}$ & Use isolators \\
\hline & $\begin{array}{l}\text { Even-order } \\
\text { Distortion }\end{array}$ & $\begin{array}{l}\text { When there are two strong } \\
\text { frequencies } \omega_{1} \text { and } \omega_{2} \text { at the } \\
\text { input of LNA, a low-frequency } \\
\text { beat } \omega_{1}-\omega_{2} \text { can be generated and } \\
\text { leak to the IF port. }\end{array}$ & Use isolators \\
\hline & $\begin{array}{l}\text { Reciprocal } \\
\text { Mixing }\end{array}$ & $\begin{array}{l}\text { Reciprocal mixing results from } \\
\text { the phase noise performance of } \\
\text { the local oscillators. }\end{array}$ & $\begin{array}{l}\text { Phase noise of } \\
\text { LO is small } \\
\text { enough to affect } \\
\text { RF signals. }\end{array}$ \\
\hline
\end{tabular}

Table 4.4 Parameters which affects performance of FMCW radar 


\begin{tabular}{|c|c|c|c|}
\hline \multirow[t]{2}{*}{ Receiver } & Noise & $\begin{array}{l}\text { It includes noise from antenna, } \\
\text { devices in receiver and spectrum } \\
\text { analyzer. }\end{array}$ & $\begin{array}{l}\text { Decrease noise in } \\
\text { receiver in future } \\
\text { work, including } \\
\text { loss of filters and } \\
\text { noise of LNA. }\end{array}$ \\
\hline & Gain & $\begin{array}{l}\text { In this radar system, receiver } \\
\text { gain is } 9.2 \mathrm{~dB}\end{array}$ & $\begin{array}{l}\text { Increase receiver } \\
\text { gain in future } \\
\text { work, including } \\
\text { gain of LNA and } \\
\text { antenna } \\
\text { efficiency. }\end{array}$ \\
\hline \multirow[t]{2}{*}{ Transmitter } & LO Leakage & $\begin{array}{l}\text { It results from finite isolation } \\
\text { between RF and LO ports of the } \\
\text { mixer. }\end{array}$ & $\begin{array}{l}\text { In this radar } \\
\text { receiver, isolation } \\
\text { of the mixer is } \\
\text { good. }\end{array}$ \\
\hline & $\begin{array}{l}\text { LO } \\
\text { Phase Noise }\end{array}$ & $\begin{array}{l}\text { Phase noise from LO may affect } \\
\text { weak RF signals. }\end{array}$ & $\begin{array}{l}\text { The phase noise of } \\
\text { LO and RF signals } \\
\text { are correlated and } \\
\text { the phase noise } \\
\text { partially cancels out. }\end{array}$ \\
\hline
\end{tabular}

This paper proposes a FMCW radar system at $2.4 \mathrm{GHz}$ for intelligent housing system. In the part of frequency source, the thesis uses ADF4158 PLL chip to generate the $2.4 \mathrm{GHz}$ continuous wave signal. It is the only PLL chip which can implement triangle frequency modulation at $2.4 \mathrm{GHz}$ band from ADI. And in the thesis, a DDS chip is used as the reference frequency of PLL. Such DDS-based PLL frequency synthesizer provides an economic and efficient method to generate frequency modulated continuous wave at ISM band in small period with high linearity. Although the thesis has some discussion about phase noise and spurs, we still need do more research on decreasing phase noise and spurs. Table 4.5 shows some problems and components which affect maximum detection range.

Table 4.5 Performances summary and comparisons

\begin{tabular}{|c|c|c|c|c|c|c|c|}
\hline Parameters & [31] & [32] & [33] & [34] & {$[35]$} & [36] & This work \\
\hline $\begin{array}{l}\text { Frequency } \\
\text { source }\end{array}$ & N/A & $\begin{array}{l}\text { DDS- } \\
\text { based } \\
\text { PLL }\end{array}$ & $\begin{array}{l}\text { NI Signal } \\
\text { Generator } \\
\text { PXI3 }\end{array}$ & $\begin{array}{l}\text { DDS- } \\
\text { based } \\
\text { PLL }\end{array}$ & NA & PLL & $\begin{array}{l}\text { DDS-based } \\
\text { PLL }\end{array}$ \\
\hline $\begin{array}{l}\text { Center } \\
\text { frequency }\end{array}$ & $\begin{array}{l}14.8 \\
\mathrm{GHz}\end{array}$ & $\begin{array}{l}122 \\
\mathrm{GHz}\end{array}$ & $5.8 \mathrm{GHz}$ & $5.8 \mathrm{GHz}$ & $\begin{array}{l}10.5 \\
\mathrm{GHz}\end{array}$ & $2.4 \mathrm{GHz}$ & $2.4 \mathrm{GHz}$ \\
\hline bandwidth & $2 \mathrm{GHz}$ & $1 \mathrm{GHz}$ & $150 \mathrm{MHz}$ & $\begin{array}{l}150 \\
\mathrm{MHz}\end{array}$ & $\begin{array}{l}0.5 \\
\mathrm{GHz}\end{array}$ & $\begin{array}{l}100 \\
\mathrm{MHz}\end{array}$ & $100 \mathrm{MHz}$ \\
\hline $\begin{array}{l}\text { Modulation } \\
\text { period }\end{array}$ & $5 \mathrm{~ms}$ & $2 \mathrm{~ms}$ & $2 \mathrm{~ms}$ & $1 \mathrm{~ms}$ & $10 \mathrm{~ms}$ & NA & $1 \mathrm{~ms}$ \\
\hline
\end{tabular}




\begin{tabular}{|c|c|c|c|c|c|c|c|}
\hline Technology & $\begin{array}{l}65 \mathrm{~nm} \\
\text { CMOS }\end{array}$ & PCB & PCB & $\begin{array}{l}\text { PCB } \\
\text { FR4 }\end{array}$ & $\begin{array}{l}0.18 \\
\text { um } \\
\text { CMOS }\end{array}$ & $\begin{array}{l}0.18 \text { um } \\
\text { CMOS }\end{array}$ & PCB FR $4^{[1]}$ \\
\hline $\begin{array}{l}\text { TX output } \\
\text { power }\end{array}$ & $9 \mathrm{dBm}$ & N/A & $13 \mathrm{dBm}$ & $10 \mathrm{dBm}$ & NA & $-7 \mathrm{dBm}$ & $23 \mathrm{dBm}$ \\
\hline RX gain & $30 \mathrm{~dB}$ & N/A & $47.5 \mathrm{~dB}$ & $40 \mathrm{~dB}$ & NA & $24 \mathrm{~dB}$ & $9.2 \mathrm{~dB}$ \\
\hline $\begin{array}{l}\text { RX noise } \\
\text { figure }\end{array}$ & $4.6 \mathrm{~dB}$ & N/A & N/A & N/A & $\begin{array}{l}11.5 \\
d B\end{array}$ & $3.5 \mathrm{~dB}$ & $3.5 \mathrm{~dB}^{[2]}$ \\
\hline Distance & $10 \mathrm{~m}$ & $2 \mathrm{~m}$ & $3 \mathrm{ft}-14 \mathrm{ft}$ & $5 \mathrm{~m}$ & $\begin{array}{l}1-7.5 \\
m\end{array}$ & $14 \mathrm{~m}$ & $0.45-10 \mathrm{~m}$ \\
\hline Average error & N/A & $2 \mathrm{~mm}$ & $0-5 \mathrm{~cm}$ & $3-4 \mathrm{~cm}$ & $4.9 \mathrm{~cm}$ & $2.14 \mathrm{~m}$ & $0.2-25 \mathrm{~cm}$ \\
\hline $\begin{array}{l}\text { Power } \\
\text { Consumption }\end{array}$ & $\begin{array}{l}210 \\
\mathrm{~mW}\end{array}$ & NA & NA & NA & NA & NA & $1.7 \mathrm{~W}$ \\
\hline
\end{tabular}

[1] We don't know what technology is used inside the DDS, PLL and mixer chip. LDMOS technology is used in power amplifier and drive amplifier in radar transmitter, and most parts in receiver, including LNA and filters are PCB.

[2] This noise figure only includes NF of receiver, doesn't include NF of power spectrum analyzer.

The comparison between the FMCW radar in this thesis and radars in other papers in shown in table 4.4. From the comparison, it can be seen that our advantages are economic and portable frequency source, and detection range is far enough for indoor position. Although we choose $2.4 \mathrm{GHz}$ ISM band for less loss in FR4 PCB, we need higher frequency has less interference, for example, bluetooth and WiFi. Paper [31] adopts $65 \mathrm{~nm}$ CMOS technology and the transmitter and receiver

To implement human tracking and positioning for intelligent housing system, the FMCW radar system needs to have three abilities:

1) the ability to determine whether people comes in or goes out;

2) the ability of positioning and tracking;

$3)$ the ability of velocity detection.

When the object moves, the power spectrum analyzer shows a fluctuation, which proves that the radar system can detect the movement of objects. According to the test shown above, the system shows the ability to detect the distance between radar and the object. As discussed in chapter 2, the velocity resolution depends on resolution bandwidth of spectrum and FMCW radar is not the best choice for velocity detection.

In conclusion, we need to make some improvements in future:

1) Improvements in device performance: I need to increase performance of LNA and BPF, decrease the noise of LNA and use material with less loss to replace 
FR-4. And we need to use antennas with high gain. We also need a new design of LNA for better input and output return loss.

2) Improvements in receiver architecture: I need to build a homodyne receiver with two orthogonal I/Q signals in order to make the results more precise.

3) Research on indoor position algorithms: Since FMCW radar can only get the distance information between radar and object, we need range-based indoor position algorithms for human positioning, for example, triangulation method.

Besides, the IF signal is small because the distance between radar and object is small. Thus, the power at $0 \mathrm{~Hz}$ caused by $\mathrm{DC}$ will influence our observation and calculation. As it has been discussed in section 2.1, FMCW radar can detect velocity information. But the Doppler frequency shift for human walking is too small, which means that it's hard to distinguish $f_{b}^{+}$and $f_{b}^{-}$from power spectrum. In order to get better range and velocity information, we can use two radars with different frequency range, which is shown in figure 4.11:

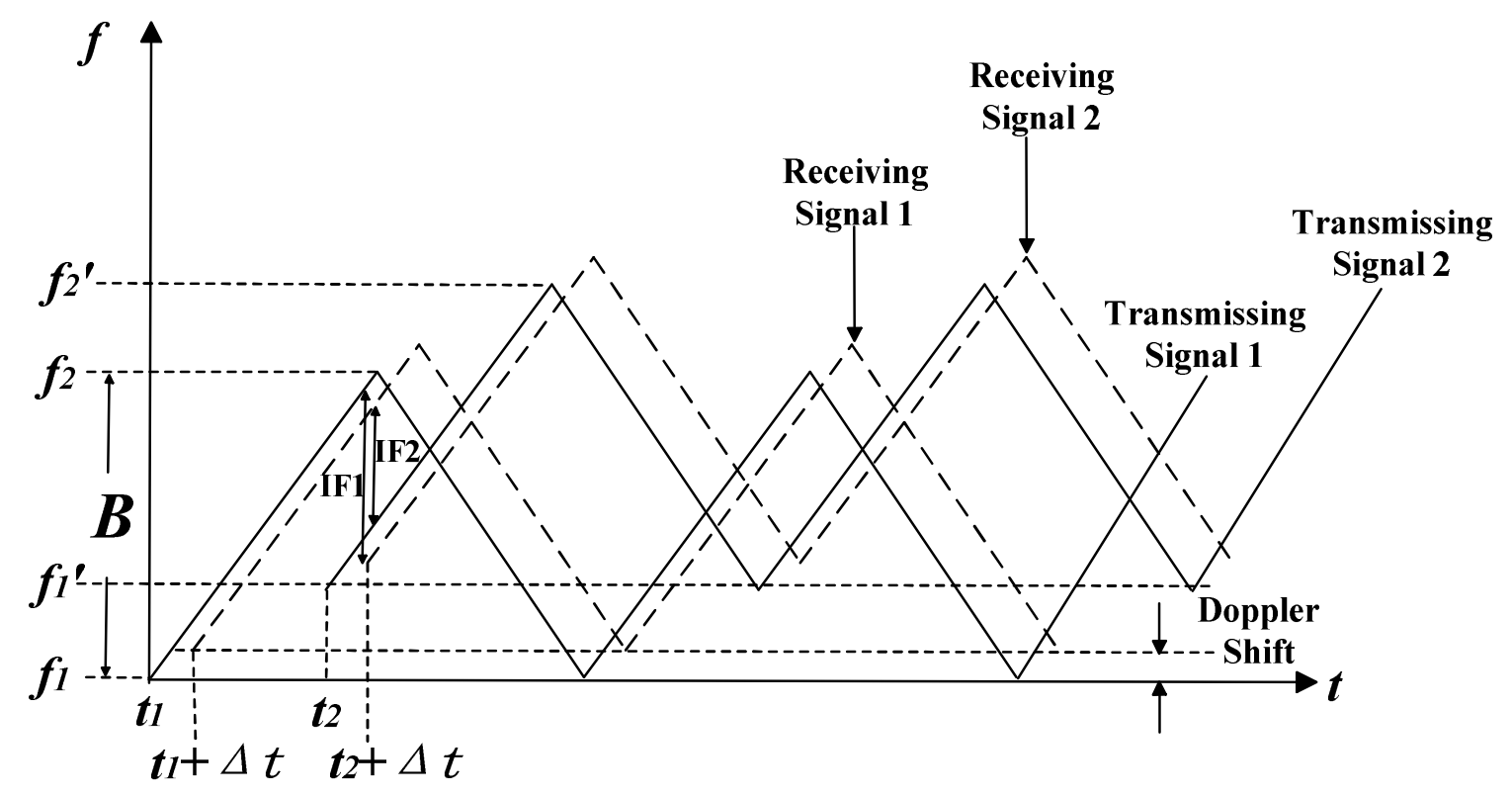

Figure 4.11 Range and velocity detection with two FMCW radars

In this radar system, $f_{1}-f_{2}=f^{\prime}{ }_{1}-f^{\prime}{ }_{2}=B$. At moment $t_{1}$, the first radar works and after $\Delta t$, the first radar gets receiving signal. At moment $t_{2}$, the second radar works. $I F_{1}$ is defined as the difference between transmitting signal 1 and receiving signal 2 , and $I F_{2}$ is defined as the difference between transmitting signal 2 and receiving signal 1. Since $t_{1}-t_{2}$ is much larger than $\Delta t, I F_{1}$ and $I F_{2}$ is much larger than $\Delta f$ discussed 
in section 2.1. Now we can move IF signal to larger frequency but we can still get $\Delta f$ :

$$
\Delta f=\frac{I F_{1}-I F_{2}}{2}
$$

Similarly, $f_{1}-f^{\prime}{ }_{1}$ is much larger than Doppler shift generated by human walking. The equation of velocity information is still the same, but at least we can observe $f_{b}^{+}$ and $f_{b}^{-}$on the power spectrum.

In conclusion, the FMCW radar system shows the ability to position and track objects in short range. And my goal is to make the system more portable, economic and effective. 


\section{Appendix}

Table 1 Range detection data

\begin{tabular}{llllll}
\hline Distance $(\mathrm{m})$ & Frequency $(\mathrm{Hz})$ & Min Error $(\mathrm{m})$ & Max Error $(\mathrm{m})$ & Average Error $(\mathrm{m})$ & SD $(\mathrm{m})$ \\
\hline 0 & 0 & 0 & 0 & 0 & 0 \\
0.45 & 600 & 0 & 0.0375 & 0.021428571 & 0.017359127 \\
0.9 & 1200 & 0 & 0.0375 & 0.026785714 & 0.013258252 \\
1.35 & 1800 & 0.01875 & 0.075 & 0.040178571 & 0.020044593 \\
1.8 & 2400 & 0.01875 & 0.09375 & 0.050892857 & 0.022410536 \\
2.25 & 3000 & 0.01875 & 0.09375 & 0.058928571 & 0.0254288 \\
2.7 & 3600 & 0.0375 & 0.1125 & 0.066964286 & 0.024421316 \\
3.15 & 4200 & 0.0375 & 0.13125 & 0.069642857 & 0.031693285 \\
3.6 & 4800 & 0.05625 & 0.13125 & 0.080357143 & 0.027332587 \\
4.05 & 5400 & 0.05625 & 0.1875 & 0.109821429 & 0.033240197 \\
4.5 & 6000 & 0.05625 & 0.13125 & 0.123214286 & 0.03808143 \\
4.95 & 6600 & 0.075 & 0.1875 & 0.128571429 & 0.034718254 \\
5.4 & 7200 & 0.09375 & 0.24375 & 0.136607143 & 0.040633584 \\
5.85 & 7800 & 0.075 & 0.1875 & 0.158035714 & 0.053033009 \\
6.3 & 8400 & 0.09375 & 0.24375 & 0.16875 & 0.045927933 \\
6.75 & 9000 & 0.075 & 0.24 & 0.173571429 & 0.050191597 \\
7.2 & 9600 & 0.1125 & 0.24375 & 0.182142857 & 0.045653734 \\
7.65 & 10200 & 0.13125 & 0.28125 & 0.211607143 & 0.051835721 \\
8.05 & 10733.33333 & 0.13125 & 0.28125 & 0.219642857 & 0.052497608 \\
8.5 & 11333.33333 & 0.13125 & 0.3 & 0.235714286 & 0.053445723 \\
8.95 & 11933.33333 & 0.15 & 0.3 & 0.241071429 & 0.04674088 \\
9.4 & 12533.33333 & 0.15 & 0.3375 & 0.265214286 & 0.057538147 \\
9.85 & 13133.33333 & 0.16875 & 0.35625 & 0.283964286 & 0.062545805 \\
10 & 13333.33333 & 0.16875 & 0.39375 & 0.308071429 & 0.072958885 \\
\hline & & & & &
\end{tabular}




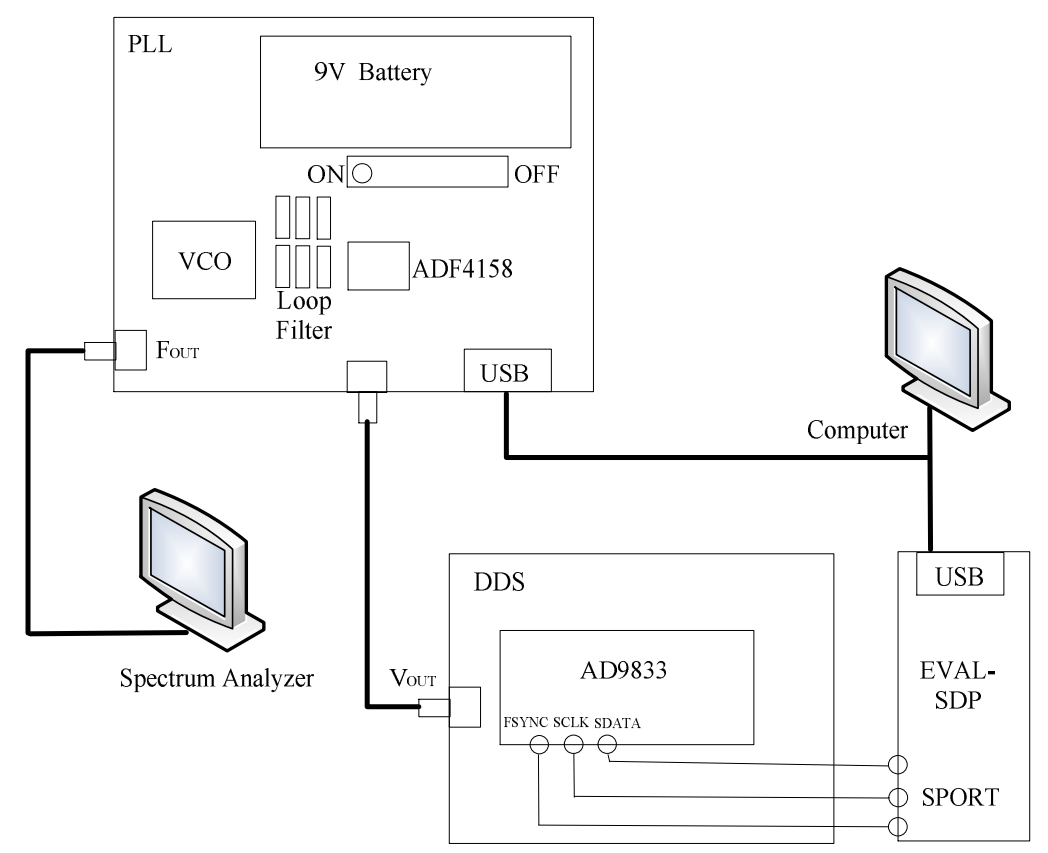

Figure 1 DDS-based PLL frequency synthesizer schematic

Table 2 DDS-based PLL Frequency Synthesizer Board Setups

\begin{tabular}{cl}
\hline Modes & Description \\
\hline RF Setups & VCO output frequency $=2.4 \mathrm{GHz}$ \\
& Reference frequency $=10 \mathrm{MHz}$ \\
& $\mathrm{R}=1 \mathrm{~N}=240$ Prescaler $=4 / 5$ \\
\hline Up Ramp & Frequency deviation per step $=40.016174 \mathrm{kHz}$ \\
& Total ramp $=100 \mathrm{MHz}$ \\
& Time per step $=0.2 \mathrm{us}$ \\
& Time per ramp $=500 \mathrm{us}$ \\
\hline Down Ramp & Frequency deviation per step $=40.016174 \mathrm{kHz}$ \\
& Total ramp $=100 \mathrm{MHz}$ \\
& Time per step $=0.2 \mathrm{us}$ \\
& Time per ramp $=500 \mathrm{us}$ \\
\hline PLL & R0=80780000 R1=1 \\
Registers & R2=8012 R3 $=443 \quad \mathrm{R} 4=180084$ \\
& R5 $=11 \mathrm{~A} 0 \mathrm{C} 85 \quad$ R6 $=4 \mathrm{E} 1 \mathrm{E} \quad \mathrm{R} 7=7$ \\
DDS & $f_{\text {out }}=12.5 \mathrm{MHz}$ \\
& Register $=8000000$ \\
\hline &
\end{tabular}




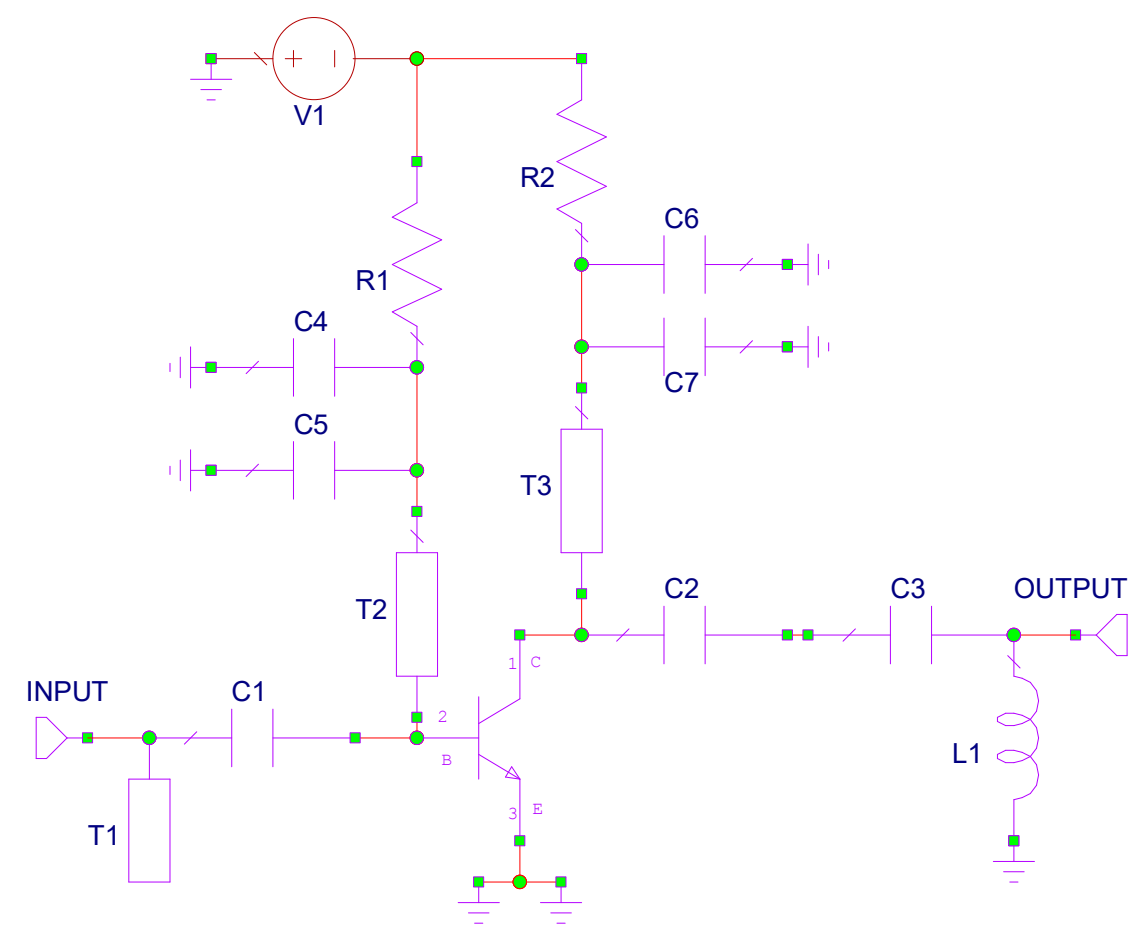

Figure 2 LNA schematic

Table 2 Bill of LNA Materials

\begin{tabular}{lll}
\hline Designator & Value & Description \\
\hline T1 & L=253.5 mil & Input Match \\
C1 & $15 \mathrm{pF}$ & DC Blocking \\
C2 & $15 \mathrm{pF}$ & DC Blocking \\
$\mathrm{C} 3$ & $1 \mathrm{pF}$ & Output Match \\
$\mathrm{L} 1$ & $4.3 \mathrm{nH}$ & Output Match \\
$\mathrm{T} 2, \mathrm{~T} 3$ & $\mathrm{~L}=657.329 \mathrm{mil}$ & LF Decoupling \\
$\mathrm{C} 4, \mathrm{C} 6$ & $1 \mathrm{e} 5 \mathrm{pF}$ & LF Decoupling \\
$\mathrm{C} 5, \mathrm{C} 7$ & $15 \mathrm{pF}$ & LF Decoupling \\
$\mathrm{R} 1$ & $18.7 \mathrm{kOhm}$ & Bias Network \\
R2 & $6 \mathrm{Ohm}$ & Bias Network \\
V1 & $3.3 \mathrm{~V}$ & \\
\hline
\end{tabular}




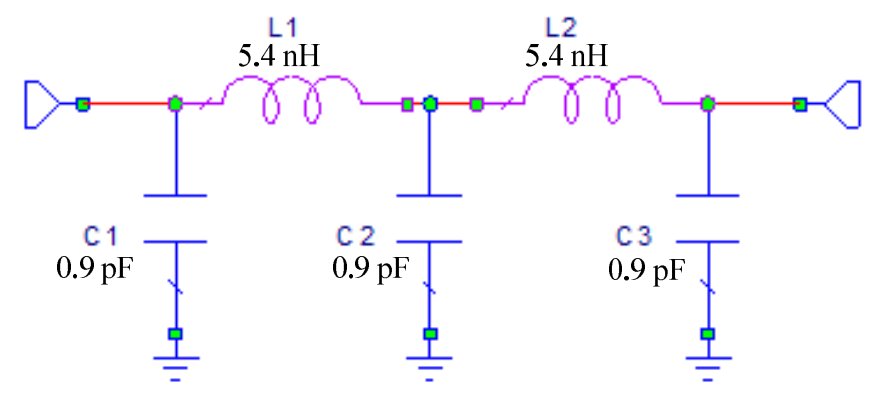

Figure 3 LPF schematic

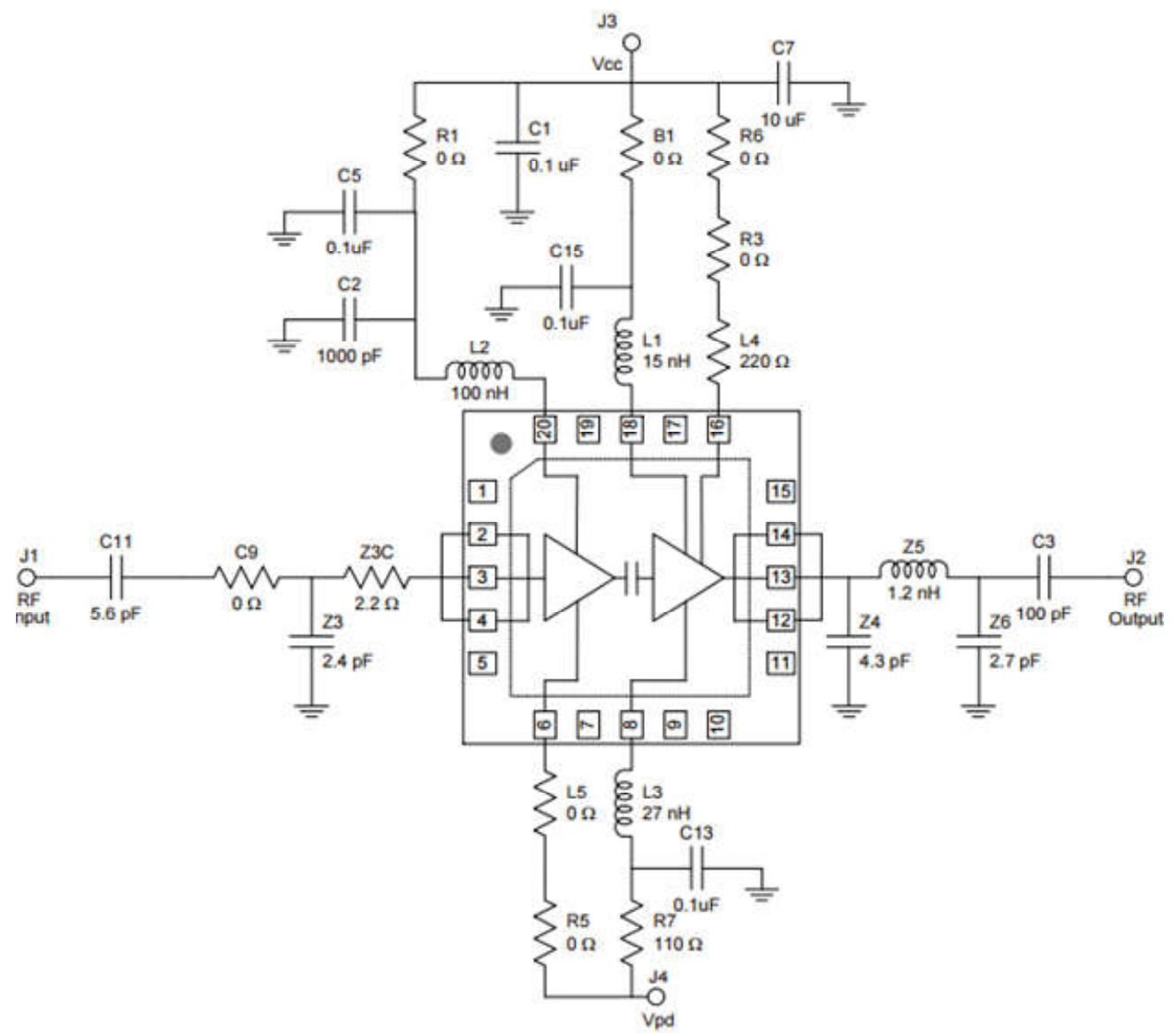

Figure 4 Power amplifier schematic 


\section{Bibliography}

[1] Mark A. Richards, "Fundamentals of Radar Signal Processing," McGraw-Hill Professional Company, pp. 1-5, 2014.

[2] Merrill I. Skolnik, "Introduction to Radar system," McGraw-Hill Professional Company, pp. 1-14, 1981.

[3] A.G. Stove, "Linear FMCW Radar Techniques," IEEE Proceedings-F, Vol. 139, No. 5, pp. 825-830, 1992.

[4] A.G. Stove, "Modern FMCW Radar - Techniques and Applications," European Radar Conference, vol. 9, no.3, pp. 149-153, 2004.

[5] Gitae Pyo, Choul-Young Kim and Songcheol Hong, "Single Antenna FMCW Radar CMOS Transceiver IC," IEEE Transactions on Microwave Theory and Techniques, vol. 2, pp. 305-310, 2016.

[6] Enxiao Liu, "The Receive and Transmit Front-end Design of X-band and Frequency Modulated Continuous Wave Radar", Dissertation Submitted to Hangzhou Dianzi University for the Degree of Master, pp. 11-12, Dec, 2012.

[7] Ronghui Zhang, "Transceiver Front-end for Short-range Target Detection", Dissertation Submitted to University of Electronic Science and Technology of China for the Degree of Master, pp. 9-11, May, 2006.

[8] Ahmed M. Eid, "System Simulation of RF Front-End Transceiver for Frequency Modulated Continuous Wave Radar", International Journal of Computer Application, vol75, pp. 16-22, Aug. 2013.

[9] Reza Sedaghati, "Analysis and Design of a Three-Phase PLL Structure", International Journal of Computer Science and Mobile Computing, Vol.3 Issue.11, pp. 385-390, Nov, 2014.

[10] Steven R. Richard, “CD4046B Types: CMOS Micropower Phase-Locked Loop,” Texas Instruments, Jul, 2003.

[11]Ahmed M. Eid, "System Simulation of RF Front-End Transceiver for Frequency Modulated Continuous Wave Radar", International Journal of Computer Application, vol75, pp. 16-22, Aug, 2013.

[12]J. Schellenberg, R. Chedester and McCoy, "Multi-Channel Receiver for an E-band FMCW Imaging Radar", IEEE/MTT-S International Microwave Symposium, pp. 1359-1362, 2013. 
[13]Eugin Hyun, Jong-Hun Lee, "A Method for Multi-target Range and Velocity Detection in Automotive FMCW Radar", Proceedings of the 12th International IEEE Conference on Intelligent Transportation System, PP. 7-11, 2009.

[14]"Direct Modulation/Waveform Generating, 6.1 GHz Fractional-N Frequency Synthesizer", pp. 26-28, Feb, 2017.

[15]A. Parssinen, J. Jussila, J. Ryynanen, L. Sumanen, K. Halonen, "A 2-GHz WideBand Direct Conversion Receiver for WCDMA Applications”, IEEE Journal of Solid-State Circuits, vol 34, pp. 1893-1903, Dec, 1999.

[16]Dennis Ma, "RF Receiver Systems and Circuits," pp. 2-13, 2014.

[17]S. Wu, B. Razavi, “A 900-MHz/1.8-GHz CMOS Receiver for Dual-Band Applications", IEEE Journal of Solid-State Circuits, vol 33, pp. 2178-2185, Dec, 1998.

[18]T. Liu, E. Westerwick, "5-GHz CMOS Radio Transceiver Front-End Chipset", IEEE Journal of Solid-State Circuits, vol 35, pp. 1927-1933, Dec, 2000.

[19]B. Razavi, "RF Microelectronics”, Upper Saddle River, pp. 153-158, 1998.

[20]Govind Singh Patel1, Sanjay Sharma, "Comparative Study of PLL, DDS and DDSbased PLL Synthesis Techniques for Communication System", International Journal of Electronics Engineering, pp. 35-40, 2010.

[21]A. Bonfanti, F. Amorosa, C. Samori, and A. L. Lacaita, "A DDS-Based PLL for 2.4-GHz Frequency Synthesis", IEEE Transactions on Circuits and Systems-ii: Analog and Digital Signal Processing, vol. 50, no. 12, Dec, 2003.

[22]B. G. Goldberg, "Digital Techniques in Frequency Synthesis”, McGraw-Hill, 1986.

[23] J. Jiang and E. K. F. Lee, “A ROM-less Direct Digital Frequency Synthesizer Using Segmented Nonlinear Digital-to-analog Converter", 2007 IEEE Region 10 Conference, vol. 5, pp. 1272-1275, 2007.

[24]Mickie P. Burt, "Range Correlation Effects in Radars", IEEE Radar Conference, Apr, 1993.

[25]H. T. Nicholas III and H. Samueli, "An analysis of the output spectrum of direct digital frequency synthesizers in the presence of phase accumulator truncation", Frequency Control Symp, pp. 495-502, 1987.

[26]Curtis Barrett, "Fractional/Integer-N PLL Basics", Texas Instruments Application Note, pp. 11-31, Aug, 1999.

[27]Russia Pascal Nelson, "Design and Analysis Frequency Synthesizers Using FPGA as Reconfiguration Hardware", Analog Devices Inc.(Greensboro, N.C.), EE Times 
Publication, pp. 45-48, Sep, 2003.

[28] Govind Singh Patel1, Sanjay Sharma, "Comparative Study of PLL, DDS and DDSbased PLL Synthesis Techniques", Communication System International Journal of Electronics Engineering, vol2(1), pp. 35-40, 2010.

[29] Saeed Golestan, Francisco D. Freijedo, Joseph M. Guerrero, “A Systematic Approach to Design Higher-Order Phase Locked Loops" IEEE Trans. Power Electronics, 2013.

[30]S. Walters, T. Troudet, "Digital Phase-Locked Loop with Jitter Bounded”, IEEE Transactions on Circuits and Systems, 36, No. 7, pp. 189- 193, July 1989.

[31]David Pozar, John Wiley, "Digital Techniques in Frequency Synthesis", McGrawHill Company, 1986.

[32]David Pozar, John Wiley, "Microwave Engineering”, McGraw-Hill Company, 2005.

[33] Yong Wang, Yuanjin Zheng, "An FMCW Radar Transceiver Chip for Object Positioning and Human Limb Motion Detection”, IEEE Sensors Journal, VOL. 17, NO. 2, Jan, 2017.

[34]Andreas Frischen, Jürgen Hasch and Christian Waldschmidt, "A Cooperative MIMO Radar Network Using Highly Integrated FMCW Radar Sensors", IEEE Transactions on Microwave Theory and Techniques, VOL. 65, NO. 4, Apr, 2017.

[35] Guochao Wang, Changzhan Gu, Takao Inoue and Changzhi Li, “A Hybrid FMCWInterferometry Radar for Indoor Precise Positioning and Versatile Life Activity Monitoring”, IEEE Transactions on Microwave Theory and Techniques, VOL. 62, NO. 11, Nov, 2014.

[36]A. Stelzer, M. Jahn and S. Scheiblhofer, “Radio and Wireless Symposium”, 2008 IEEE Radio and Wireless Symposium, Mar, 2008.

[37] Silvan Wehrli, David Barras, Frank Ellinger and Heinz Jackel, "Integrated Active Pulsed Reflector for FMCW Radar Localization”, 2009 IEEE MTT-S International Microwave Symposium Digest, Jul, 2009.

[38]Niko Joram, Belal Al-Qudsi, Jens Wagner, Axel Strobel and Frank Ellinger, "Design of a Multi-Band FMCW Radar Module", 2013 10th Workshop on Positioning, Navigation and Communication (WPNC), Mar, 2013. 